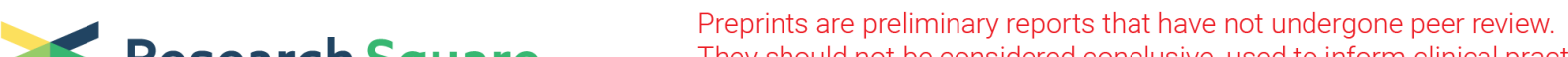 $\begin{array}{ll}\text { Research Square } & \text { They should not be considered conclusive, used to inform clinical practice, } \\ \text { or referenced by the media as validated information. }\end{array}$ \\ Evaluation of clinical value and potential mechanism of MTFR2 in lung adenocarcinoma via bioinformatics
}

\section{Cheng Chen}

Department of Thoracic Surgery, Affiliated Hospital of Zunyi Medical University, Zunyi 563000, Guizhou, China

\section{Yang Tang}

Department of Thoracic Surgery, Affiliated Hospital of Zunyi Medical University, Zunyi 563000, Guizhou, China

\section{Wendong Qu}

Department of Thoracic Surgery, Affiliated Hospital of Zunyi Medical University, Zunyi 563000, Guizhou, China

\section{Xu Han}

Department of Thoracic Surgery, Affiliated Hospital of Zunyi Medical University, Zunyi 563000, Guizhou, China

Jiebin Zuo

Department of Thoracic Surgery, Affiliated Hospital of Zunyi Medical University, Zunyi 563000, Guizhou, China

\section{Qingyong Cai}

Department of Thoracic Surgery, Affiliated Hospital of Zunyi Medical University, Zunyi 563000, Guizhou, China

\section{Gang Xu}

Department of Thoracic Surgery, Affiliated Hospital of Zunyi Medical University, Zunyi 563000, Guizhou, China

\section{Yongxiang Song ( $\nabla$ songtang2004@163.com )}

Department of Thoracic Surgery, Affiliated Hospital of Zunyi Medical University, Zunyi 563000, Guizhou, China

\section{Xixian Ke ( $\square$ kexixian@zmu.edu.cn )}

Department of Thoracic Surgery, Affiliated Hospital of Zunyi Medical University, Zunyi 563000, Guizhou, China

\section{Research Article}

Keywords: lung adenocarcinoma, prognosis, MTFR2, bioinformatics, biomarker 
Posted Date: December 10th, 2020

DOI: https://doi.org/10.21203/rs.3.rs-118595/v1

License: (c) (i) This work is licensed under a Creative Commons Attribution 4.0 International License. Read Full License 


\section{Abstract \\ Background}

Mitochondrial fission regulator 2 (MTFR2) was involved in the progression and development of various cancers. However, the relationship between MTFR2 with lung adenocarcinoma (LUAD) had not been reported. Herein, this study analyzed the clinical significance and potential mechanisms of MTFR2 in LUAD via bioinformatic tools.

\section{Results}

We found that the level of MTFR2 was increased, and correlated with sex, age, smoking history, neoplasm staging, histological subtype and TP53 mutation status in LUAD patients. Kaplan-Meier survival analysis showed LUAD patients with increased MTFR2 had a poor prognosis. In addition, univariate COX regression analysis showed neoplasm staging, T stage, distant metastasis and MTFR2 level were risk factors for the prognosis of LUAD. A total of 1127 genes were coexpressed with MTFR2, including 840 positive and 208 negative related genes. KEGG and GSEA found that MTFR2 participated in the progression of LUAD by affecting cell cycle, DNA replication, homologous recombination, p53 signaling pathway and other mechanisms. The top 10 coexpressed genes, namely CDK1, CDC20, CCNB1, PLK1, CCNA2, AURKB, CCNB2, BUB1B, MAD2L1 and BUB1 were highly expressed, and were associated with poor prognosis in LUAD.

\section{Conclusions}

Consequently, we elucidated MTFR2 was a biomarker for diagnosis and poor prognosis in LUAD, and might participate in the progression of LUAD via affecting cell cycle, DNA replication, homologous recombination and p53 signaling pathway.

\section{Introduction}

Non-small cell lung cancer (NSCLC) was one of the most common malignant tumors in the world, and lung adenocarcinoma (LUAD) was one of the common subtypes of NSCLC [1, 2]. LUAD patients in early stage got improved long-term prognosis by surgery and neoadjuvant chemotherapy, while due to incomplete excision, primary and secondary drug resistance and other reasons, recurrence and metastasis happened. The 5-year survival rate of the patients was still very low, and its treatment was extremely challenging $[2,3]$. In recent years, targeted therapy had greatly improved the prognosis of patients with LUAD, but the mortality of patients with LUAD remained at a high level [4-6]. Therefore, it was of great significance to further discover the novel molecules and explore their regulatory mechanisms involved in the occurrence and development of LUAD resulting in finding novel therapeutic targets. 
Mitochondrial fission regulator 2 (MTFR2), also known as FAM54A, was located on the 6q23.2 chromosome and belonged to the MTFR family. Abnormal mitosis of mitochondria was related to the pathogenesis of many diseases, and it promoted the development, and was correlated to occurrence and prognosis of several tumors [7-11]. MTFR2 was up-regulated in breast cancer and was related to age, lymph node metastasis, and prognosis of HER2 positive breast cancer patients. Knockout of MTFR2 prohibited the proliferation, migration and invasion of breast cancer cells, and blocked epithelial-stromal transformation (EMT) [8]. MTFR2 was related to the transcriptional regulation of TTK expression by activating the promoter. MTFR2-dependent TTK regulation played a key role in maintaining GSC (Glioma stem-like cells) in glioma, and may be the target molecule of new drugs for glioma patients [12]. Whereas, at present, rare researches were focused on MTFR2 in other tumors including LUAD, except for those cancers listed above. Herein, the present study explored the clinical value and potential regulatory mechanism of MTFR2 in the progression of LUAD through multiple databases.

\section{Results}

\subsection{MTFR2 was upregulated and correlated with sex, age, smoking history, cancer stage, histological subtype and TP53 mutation status in LUAD patients}

MTFR2 was abnormally expressed in pan-cancerous tissues, and was mainly over-expressed in a variety of tumor tissues (Fig 1). In detail, in the Oncomine database, MTFR2 was highly expressed in breast cancer, colorectal cancer, gastric cancer, lung cancer and other tumor tissues, while lowly expressed in brain nerve tumor, breast cancer, leukemia and other tumor tissues (Fig 1A). In Timer database, MTFR2 was highly expressed in BLCA, BRCA, CHOL, LUSC, LUAD and other tumor tissues (Fig 1B).

In addition, we found similar results in the Ualcan database, where MTFR2 was highly expressed in LUAD tissues (Table 1). In addition, in the TCGA database, the expression of MTFR2 was increased in unpaired LUAD patients (Fig 2A) and in 57 pairs of LUAD patients (Fig 2B).

In the Ualcan database, we found that the expression of MTFR2 was related to the clinicopathological features of LUAD patients (Table 1). In detail, the level of MTFR2 was related to the sex (Male vs Female), Age (Age(41-60Yrs) vs Age (61-80Yrs)), Smoking (Non smoker vs Smoker; Non smoker vs Reformed smoker2; Smoker vs Reformed smoker1; Smoker vs Reformed smoker2; Reformed smoker1 vs Reformed smoker2), histological subtype (NOS vs Mixed; NOS vs LBC-Non Mucinous; NOS vs Solid Pattern Predominant; NOS vs Acinar; NOS vs LBC-Mucinous; NOS vs Papillary; Mixed vs Solid Pattern Predominant; Mixed vs LBC-Mucinous; Mixed vs Papillary; Clear Cell vs LBC-Non Mucinous; Clear Cell vs Papillary; LBC-Non Mucinous vs Solid Pattern Predominant; LBC-Non Mucinous vs LBC-Mucinous; Solid Pattern Predominant vs LBC-Mucinous; Solid Pattern Predominant vs Mucinous carcinoma; Solid Pattern Predominant vs Papillary; Acinar vs LBC-Mucinous; LBC-Mucinous vs Papillary) and TP53 mutation (TP53-Mutant vs TP53-Non Mutant) of LUAD patients. 
In TCGA database, ROC analysis showed that the level of MTFR2 had a diagnostic value in LUAD patients (Figs 2C and D). Further Kaplan-Meier analysis showed that the LUAD patients with increased MTFR2 had a poor prognosis (Fig 2E). In addition, in the Kaplan-Meier Plotter database, the expression levels of MTFR2 (228069_at) and MTFR2 (234944_at) were associated with the overall survival and early disease progression of LUAD patients (Figs 2F-I). Univariate Cox analysis of complete clinical data of LUAD patients in TCGA database showed that cancer stage, T stage, distant metastasis as well as MTFR2 expression were risk factors for the prognosis of LUAD patients (Table 2). Further multivariate Cox analysis showed that cancer stage was an independent risk factor for the prognosis of LUAD patients (Table 2).

\subsection{Screening of MTFR2 co-expressed genes}

We screened 1127 MTFR2 co-expressed genes in TCGA database by screening conditions, most of which were positive related genes. Among them, there were 840 positive and 208 negative related genes (S1 Table ). The top 10 genes that were positively and negatively related to MTFR2 (Table 3). The expression of the top 10 positive and negative related genes of MTFR2 in LUAD tissues from TCGA were abnormally expressed (Fig 3).

\subsection{Analysis of MTFR2 and its coexpressed genes by GO, KEGG and GSEA}

In order to further understand the potential function of MTFR2 in the development of LUAD, we carried out GO and KEGG analysis of MTFR2 co-expressed genes. MTFR2 coexpressed genes were involved in biological processes (BP), cellular component (CC) and molecular function (MF) (Figs 4A-C), and in details, were mainly involved in DNA replication, chromosome segregation, mitotic nuclear division, regulation of mitotic cell cycle phase transition and other processes (S2 Table). KEGG pathway analysis indicated MTFR2 co-expressed genes were mainly involved in cell cycle, DNA replication, proteasome, homologous recombination, spliceosome, nucleotide excision repair, human T-cell leukemia virus 1 infection, p53 signaling pathway and other signal pathways (Fig 4D and Table 4). Secondly, GSEA found that cell cycle, DNA_replication, homologous recombination, P53 signal pathway, oocyte meiosis and base_excision repair signaling pathways were highly enriched in MTFR2 highly expressed group (Fig 5 and Table 5). To sum up, these results suggested that MTFR2 might regulate the progress of LUAD through cell cycle, DNA replication, Homologous recombination and P53 signaling pathway.

\subsection{Construction of PPI network and the expressions of Hub genes and its prognostic value}


By identifying the function of MTFR2 co-expressed genes, the potential biological function of MTFR2 was inferred. The top 10 Hub genes in the PPI network were selected by the CytoHubba plug-in as CDK1, CDC20, CCNB1, PLK1, CCNA2, AURKB, CCNB2, BUB1B, MAD2L1 and BUB1 (S Fig and Table 6). The expression of MTFR2 was correlated with that of CDK1, CDC20, CCNB1, PLK1, CCNA2, AURKB, CCNB2, BUB1B, MAD2L1 and BUB1 (Fig 6). In addition, GEPIA online website was used to analyze the expressions of Hub genes and its prognostic value. We found that the expressions of CDK1, CDC20, CCNB1, PLK1, CCNA2, AURKB, CCNB2, BUB1B, MAD2L1 and BUB1 genes were significantly increased in LUAD tissues (Fig 7). The top10 Hub genes were associated with overall survival in LUAD patients, and CDK1, CCNB1, PLK1, AURKB, CCNB2, BUB1B and BUB1 were associated with disease free survival (DFS) in LUAD patients with disease-free progression (Fig 8). These results further indicated that MTFR2 played an vital role in the diagnosis, treatment and disease progression of LUAD.

\section{Discussion}

Mitochondria, via regulating the membrane potential, participated in programmed cell death as well as cell proliferation $[13,14]$. MTFR2 played an vital role in promoting the division of mitochondria in cells. Under pathological condition, abnormal mitosis of mitochondria was related to the development of tumors $[10,11]$. At present, studies found that the expression of MTFR2 in breast cancer tissue was increased, and was correlated to the clinicopathological features and poor prognosis of patients. Interfering with MTFR2 inhibited the growth and migration of breast cancer cells, indicating its biological role as oncogene and with prognostic value [8]. However, the role of MTFR2 in LUAD was still not been elucidated. In present study, it was found for the first time that MTFR2 was highly expressed in LUAD tissues in multiple databases, and the level of MTFR2 was related to sex, age, smoking history, cancer stage, histological subtypes and TP53 mutation status in LUAD patients. ROC analysis showed that the expression of MTFR2 had diagnostic value in LUAD. Kaplan-Meier analysis showed that the overall survival time of LUAD patients with increased MTFR2 expression was related to the early disease progression. Univariate Cox analysis showed that cancer stage, $T$ stage, distant metastasis and the level MTFR2 were risk factors for the prognosis in LUAD. These results indicated that MTFR2 played an important role in the development of LUAD.

Abnormal cell cycle leaded to abnormal regulation of cell growth and induced overgrowth of cells, resulting in poor prognosis of tumor patients. Therefore, through a detailed understanding of the mechanism of tumor growth and inhibition of tumor occurrence and development, the prognosis of patients could be improved [15-17]. For example, the expression of DMBX1 (Diencephalon/ mesencephalon homeobox 1) in LUAD tissue was increased, and was related to the poor prognosis and clinicopathological features of LUAD patients [18]. Silencing of DMBX1 inhibited the proliferation of LUAD and induced $\mathrm{G} 1$ to $S$ phase arrest, while overexpression of DMBX1 enhanced the growth and the transformation from $\mathrm{G} 1$ to S phase. DMBX1 inhibited the expression of OTX2 and p21 transcription to participate in the progression of LUAD [18]. Ailanthone (Aila) inhibited the growth of non-small cell lung cancer (NSCLC) in vitro and in vivo, and prolonged the survival time of tumor-bearing mice. And Aila induced cell cycle arrest in a dose-dependent manner. In addition, Aila inhibited DNA replication by down- 
regulating replication protein A1 (RPA1), thus inhibiting the growth of NSCLC [19]. Nichols et al. reported that high mutation burden in LUAD patients with increased HORMAD1 expression decreased the survival rate of LUAD patients. HORMAD1 was one of the key genes in the process of homologous recombination $(\mathrm{HR})$, and promoted the formation of RAD51 filaments to participate in the process of homologous recombination. Interfering with HORMAD1 reduced the growth of tumor cells [20]. FAM111B was highly expressed in LUAD tissues. LUAD patients with high expression of FAD111B had low relapse-free survival (RFS) and overall survival (OS). Interfering with FAM111B inhibited the proliferation, cell cycle arrest and migration of LUAD cells and the ability of tumor formation in nude mice, but induced apoptosis. FAM111B might regulate the growth of LUAD through p53 signaling pathway, inhibit the expression of BAG3 and BCL2, and play an important role in cell cycle and apoptosis [21]. In present study, we found that MTFR2 coexpressed genes were mainly involved in cell cycle, DNA replication, homologous recombination, p53 signaling pathway and other signaling pathways by KEGG. Secondly, GSEA also found that cell cycle, DNA replication, homologous recombination and p53 signaling pathways were highly enriched in MTFR2 high expression group. To date, it was found that interfering with MTFR2 regulated the process of EMT [8], but the mechanism related to the growth of LUAD was still not been elucidated. However, MTFR2 promoted mitochondrial division in cells, which was related to tumor progression. Therefore, we speculated that MTFR2 might regulate the progress of LUAD through cell cycle, DNA replication, homologous recombination and p53 signaling pathways.

Differential expression of genes in the process of tumorigenesis and development, regulated the progression of cancers and affected the prognosis of patients [22-25]. Polo-likekinase1 (PLK1) was a key regulatory factor in cell cycle and apoptosis. PLK1 was overexpressed in many kinds of Cutaneous Tcell lymphomas (CTCL) cells. Down-regulation of PLK1 by knockdown or inhibitors GW843682X inhibited cell growth, stability and proliferation, and also leaded to cell cycle arrest, mitotic protein changes, apoptosis and mitotic abnormalities [22]. AZD5438, cyclin-dependent kinase (CDK) inhibitors, enhanced radiosensitivity by inhibiting CDK1, prolonging the block of $\mathrm{G} 2$ to $\mathrm{M}$ stage, inhibiting homologous recombination (HR), delaying DNA repair and increasing apoptosis in NSCLC cells [23]. CCNA2 was highly expressed in NSCLC, and the prognosis of patients with high expression of it was worse than that of patients with low expression [24]. Topoisomerase IIA (TOP2A) was highly expressed in LUAD, and was associated with poor prognosis, indicating that TOP2A was a prognostic marker in LUAD. Knockdown of TOP2A in A549 and GLC82 cells inhibited cell proliferation, migration and invasion, and decreased the expression of CCNB1 and CCNB2, indicating that targeting CCNB1 and CCNB2 promoted the progress of LUAD [25]. In this study, we found that the hub genes were involved in a variety of signal pathways including in the occurrence and development of tumors. GEPIA online analysis of hub genes in PPI network showed that the expressions of CDK1, CDC20, CCNB1, PLK1, CCNA2, AURKB, CCNB2, BUB1B, MAD2L1 and BUB1 were increased in LUAD and were related to the overall survival (OS), while CDK1, CCNB1, PLK1, AURKB, CCNB2, BUB1B and BUB1 were associated with disease-free survival (DFS) in LUAD patients. The expression of MTFR2 was also closely related to that of CDK1, CDC20, CCNB1, PLK1, CCNA2, AURKB, CCNB2, BUB1B, MAD2L1 and BUB1, which further indicated that MTFR2 played an vital 
role in the diagnosis, treatment and disease progression of LUAD. However, the regulatory mechanism between Hub gene and MTFR2 was not clear, which needed to be further verified.

Generally speaking, we found that the high expression of MTFR2 was related to the clinicopathological features, diagnosis and prognosis in LUAD patients, and it might be used as a potential target for diagnosis and prognosis, and promote the occurrence and development of LUAD through cell cycle, DNA replication, homologous recombination and p53 signal pathway.

\section{Materials And Methods}

\subsection{Oncomine and Timer database}

The level of MTFR2 in pan-cancer tissues was analyzed by Oncomine database (https:// www.oncomine.org/resource/login) and Timer database (https://cistrome. shinyapps.io/timer/). Oncomine database screening criteria: (1) Gene: FAM54A; (2) Analysis Type: Cancer vs Normal; (3) Date Type: $m R N A$; (4) $P<0.05$; (5) Fold Chang $\geq 1.5$. Search for FAM54A in the gene module of Timer database to analyze its level in pan-cancer tissues.

\subsection{Ualcan database}

Ualcan database (http://ualcan.path.uab.edu) was used to explore the expression of MTFR2 in LUAD tissues, and the correlation between the level of MTFR2 with the clinicopathological charicteristics (including sex, age, race, smoking history, stage, histological subtype and so on) of LUAD patients was analyzed.

\subsection{TCGA and Kaplan-Meier Plotter database}

The gene expression data of 594 cases of LUAD with HTSeq-FPKM were downloaded from the official website of TCGA, including 59 cases of normal lung tissues and 535 cases of LUAD tissues. Among them, the gene expression data of 57 normal lung tissues and 57 LUAD tissues came from the same patient. TCGA data were used to analyze the expression of MTFR2 in LUAD tissues and its diagnostic value. The clinical data of 522 patients with LUAD were downloaded and screened. The patients with unknown or incomplete clinical characteristic information and lack of prognostic follow-up data were excluded, and the complete clinical data were retained for prognostic correlation analysis. The data of Kaplan-Meier Plotter database (http://kmplot.com/analysis/) were derived from RNA data of TCGA and GEO databases. According to the median value of MTFR2 mRNA level, the patients were divided into two groups: high and low expression groups to explore the correlation between its level with the prognosis of LUAD patients. 


\subsection{Screening of MTFR2 co-expressed genes}

Pearson coefficient $(r)$ was applied to indicate the correlation between genes and reflect the biological relationship between the two genes. $R$ was used to screen MTFR2 coexpressed genes in 535 cases of LUAD tissues from TCGA. Screening criteria: $P<0.001$ and Pearson coefficient $(r>0.4$ or $r<-0.4)$, which were defined as moderate or above MTFR2 co-expressed genes.

\subsection{GO, KEGG and GSEA}

The co-expressed genes of MTFR2 were analyzed by GO and KEGG using R to explore the biological function and related signal pathways of LUAD which may be involved in MTFR2. The gene expression data of 535 patients with LUAD from TCGA were divided into high and low expression groups according to the median MTFR2 expression level. The genes were arranged according to the degree of differential expression in the two kinds of samples by GSEA to explore the effect of MTFR2 level on each gene. Each analysis was repeated 1000 times. Screening criteria: NOM $p<0.05$.

\subsection{PPI network construction}

STRING (https://string-db.org/) database was used to analyze the protein-protein interaction of multiple genes. We introduced MTFR2 co-expressed genes into STRING database to construct PPI network. The screening condition: combined score $>0.9$ was considered to be statistically significant. The obtained PPI network was introduced into Cytoscape3.6.1 software, and the top 10 genes with high connectivity were screened by CytoHubba plug-in, and they were defined as Hub genes.

\subsection{GEPIA database}

The LUAD data from TCGA and GTEx databases and RNA data from normal tissues were selected from GEPIA (Gene Expression Profiling Interactive Analysis) database to verify the expression of Hub gene and to analyze whether there was differential expression between cancer and normal tissues. According to the median value of Hub gene expression, the patients were divided into two groups: high and low expression groups, and the relationship between its expression level and overall survival (OS) and disease-free survival (DFS) in patients with LUAD was analyzed.

\section{Abbreviations}

BP, biological processes;

CC, cellular component;

MF, molecular function; 
OS, overall survival;

DFS, disease-free survival;

GEPIA, Gene Expression Profiling Interactive Analysis;

MTFR2, Mitochondrial fission regulator 2

LUAD, lung adenocarcinoma

NSCLC, Non-small cell lung cancer

EMT, epithelial-stromal transformation

GSC, Glioma stem-like cells

MF, molecular function

RPA1, replication protein A1

$H R$, homologous recombination

RFS, relapse-free survival

PLK1, Polo-likekinase1

CTCL, Cutaneous T-cell lymphomas

CDK, cyclin-dependent kinase

\section{Declarations}

\section{Ethics approval and consent to participate}

This manuscript does not involve animal and human experiments, so it has not yet applied the Ethics approval and consent to participate.

\section{Consent for publication}

Not applicable.

\section{Availability of data and materials}

Publicly available datasets were analyzed in this study. The datasets generated for this study could be found in TCGA (https://portal.gdc.cancer.gov/projects/TCGA-LUAD (HTSeq-FPKM)).

\section{Competing interests}


The authors declare no conflicts of interests.

\section{Funding}

This work was supported by Grants from the National Natural Science Foundation of China (No. 81960532).

\section{Authors' contributions}

This research was finished by all the authors. SYX conceived the research topic, made the research plan and directed the implementation of the whole research. KXX drafted the manuscript together and processed the data. CC, XG, CQY and TY assisted to collect and analyze the data and draft the manuscript. QWD, HX and ZJB were mainly involved in the management of lung cancer samples and related data collection. All authors read and approved the final manuscript.

\section{Acknowledgments}

Thanks to the thoracic surgeons of Zunyi Medical University for their assistance in collecting lung cancer specimens.

\section{Authors' information}

${ }^{1}$ Department of Thoracic Surgery, Affiliated Hospital of Zunyi Medical University, Zunyi 563000, Guizhou, China

\section{References}

1. Song $Y$, Chen $D$, Zhang $X$, Luo Y, Li S: Integrating genetic mutations and expression profiles for survival prediction of lung adenocarcinoma. Thoracic cancer 2019, 10(5):1220-1228.

2. Zhou W, Liu Y, Gao Y, Cheng Y, Chang R, Li X, Zhou Y, Wang S, Liang L, Duan C et al: MICAL2 is a novel nucleocytoplasmic shuttling protein promoting cancer invasion and growth of lung adenocarcinoma. Cancer letters 2020, 483:75-86.

3. Mizutani K, Guo X, Shioya A, Zhang J, Zheng J, Kurose N, Ishibashi H, Motono N, Uramoto H, Yamada $\mathrm{S}$ : The impact of PRDX4 and the EGFR mutation status on cellular proliferation in lung adenocarcinoma. International journal of medical sciences 2019, 16(9):1199-1206.

4. Okita R, Maeda A, Shimizu K, Nojima Y, Saisho S, Nakata M: PD-L1 overexpression is partially regulated by EGFR/HER2 signaling and associated with poor prognosis in patients with non-smallcell lung cancer. Cancer immunology, immunotherapy: C/I 2017, 66(7):865-876.

5. Khunger M, Jain P, Rakshit S, Pasupuleti V, Hernandez AV, Stevenson J, Pennell NA, Velcheti V: Safety and Efficacy of PD-1/PD-L1 Inhibitors in Treatment-Naive and Chemotherapy-Refractory Patients With Non-Small-Cell Lung Cancer: A Systematic Review and Meta-Analysis. Clinical lung cancer 2018, 19(3):e335-e348. 
6. Jan YH, Lai TC, Yang CJ, Huang MS, Hsiao M: A co-expressed gene status of adenylate kinase 1/4 reveals prognostic gene signature associated with prognosis and sensitivity to EGFR targeted therapy in lung adenocarcinoma. Scientific reports 2019, 9(1):12329.

7. Feng J, Ma Y, Chen Z, Hu J, Yang Q, Ding G: Mitochondrial pyruvate carrier 2 mediates mitochondrial dysfunction and apoptosis in high glucose-treated podocytes. Life sciences 2019, 237:116941.

8. Lu G, Lai Y, Wang T, Lin W, Lu J, Ma Y, Chen Y, Ma H, Liu R, Li J: Mitochondrial fission regulator 2 (MTFR2) promotes growth, migration, invasion and tumour progression in breast cancer cells. Aging 2019, 11(22):10203-10219.

9. Wu Y, Chen $\mathrm{M}$, Jiang J: Mitochondrial dysfunction in neurodegenerative diseases and drug targets via apoptotic signaling. Mitochondrion 2019, 49:35-45.

10. Zhou Q, Li H, Li Y, Tan M, Fan S, Cao C, Meng F, Zhu L, Zhao L, Guan MX et al: Inhibiting neddylation modification alters mitochondrial morphology and reprograms energy metabolism in cancer cells. JCl insight 2019, 4(4).

11. Zeng Y, Guo Z, Hu Z, Liu M, Chen Y, Chen S, Peng B, Zhang P, Wu Z, Luo H et al: FGD1 exhibits oncogenic properties in hepatocellular carcinoma through regulating cell morphology, autophagy and mitochondrial function. Biomedicine \& pharmacotherapy = Biomedecine \& pharmacotherapie 2020, 125:110029.

12. Wang J, Xie Y, Bai X, Wang N, Yu H, Deng Z, Lian M, Yu S, Liu H, Xie W et al: Targeting dual specificity protein kinase TTK attenuates tumorigenesis of glioblastoma. Oncotarget 2018, 9(3):3081-3088.

13. Yang $M$, Wang B, Gao J, Zhang Y, Xu W, Tao L: Spinosad induces programmed cell death involves mitochondrial dysfunction and cytochrome $C$ release in Spodoptera frugiperda Sf 9 cells. Chemosphere 2017, 169:155-161.

14. Yang S, Liu Y, Guo Y, Liu R, Qi F, Li X, Yu H, Cheng S, Wang Z: Circadian gene Clock participates in mitochondrial apoptosis pathways by regulating mitochondrial membrane potential, mitochondria out membrane permeablization and apoptosis factors in AML12 hepatocytes. Molecular and cellular biochemistry 2020, 467(1-2):65-75.

15. Pan B, Zhong W, Deng Z, Lai C, Chu J, Jiao G, Liu J, Zhou Q: Inhibition of prostate cancer growth by solanine requires the suppression of cell cycle proteins and the activation of ROS/P38 signaling pathway. Cancer medicine 2016, 5(11):3214-3222.

16. Yue M, Li S, Yan G, Li C, Kang Z: Paeoniflorin inhibits cell growth and induces cell cycle arrest through inhibition of FoxM1 in colorectal cancer cells. Cell cycle (Georgetown, Tex) 2018, 17(2):240249.

17. Li W, Zheng G, Xia J, Yang G, Sun J, Wang X, Wen M, Sun Y, Zhang Z, Jin F: Cell cycle-related and expression-elevated protein in tumor overexpression is associated with proliferation behaviors and poor prognosis in non-small-cell lung cancer. Cancer science 2018, 109(4):1012-1023.

18. Luo J, Liu K, Yao Y, Sun Q, Zheng X, Zhu B, Zhang Q, Xu L, Shen Y, Ren B: DMBX1 promotes tumor proliferation and regulates cell cycle progression via repressing OTX2-mediated transcription of p21 in lung adenocarcinoma cell. Cancer letters 2019, 453:45-56. 
19. Ni Z, Yao C, Zhu X, Gong C, Xu Z, Wang L, Li S, Zou C, Zhu S: Ailanthone inhibits non-small cell lung cancer cell growth through repressing DNA replication via downregulating RPA1. British journal of cancer 2017, 117(11):1621-1630.

20. Nichols BA, Oswald NW, McMillan EA, McGlynn K, Yan J, Kim MS, Saha J, Mallipeddi PL, LaDuke SA, Villalobos PA et al: HORMAD1 Is a Negative Prognostic Indicator in Lung Adenocarcinoma and Specifies Resistance to Oxidative and Genotoxic Stress. Cancer research 2018, 78(21):6196-6208.

21. Sun H, Liu K, Huang J, Sun Q, Shao C, Luo J, Xu L, Shen Y, Ren B: FAM111B, a direct target of p53, promotes the malignant process of lung adenocarcinoma. OncoTargets and therapy 2019, 12:28292842.

22. Nihal M, Stutz N, Schmit T, Ahmad N, Wood GS: Polo-like kinase 1 (Plk1) is expressed by cutaneous T-cell lymphomas (CTCLs), and its downregulation promotes cell cycle arrest and apoptosis. Cell cycle (Georgetown, Tex) 2011, 10(8):1303-1311.

23. Raghavan P, Tumati V, Yu L, Chan N, Tomimatsu N, Burma S, Bristow RG, Saha D: AZD5438, an inhibitor of Cdk1, 2, and 9, enhances the radiosensitivity of non-small cell lung carcinoma cells. International journal of radiation oncology, biology, physics 2012, 84(4):e507-514.

24. Ko E, Kim Y, Cho EY, Han J, Shim YM, Park J, Kim DH: Synergistic effect of Bcl-2 and cyclin A2 on adverse recurrence-free survival in stage I non-small cell lung cancer. Annals of surgical oncology 2013, 20(3):1005-1012.

25. Kou F, Sun H, Wu L, Li B, Zhang B, Wang X, Yang L: TOP2A Promotes Lung Adenocarcinoma Cells' Malignant Progression and Predicts Poor Prognosis in Lung Adenocarcinoma. Journal of Cancer 2020, 11(9):2496-2508.

\section{Tables}

Table 1. The expression of MTFR2 in Ualcan database was correlated to the clinicopathological characteristics in LUAD patients. 


\begin{tabular}{|c|c|}
\hline Clinicopathological characteristics & $p$ value \\
\hline \multicolumn{2}{|l|}{ Expression } \\
\hline Normal-vs-Primary & $1.62447832963153 \mathrm{E}-12$ \\
\hline \multicolumn{2}{|l|}{ Gender } \\
\hline Male-vs-Female & 4.185800E-02 \\
\hline \multicolumn{2}{|l|}{ Age } \\
\hline Age(41-60Yrs)-vs-Age(61-80Yrs) & $1.089890 \mathrm{E}-02$ \\
\hline \multicolumn{2}{|l|}{ Smoking } \\
\hline Non smoker-vs-Smoker & 7.3849000004067E-07 \\
\hline Non smoker-vs-Reformed smoker2 & $6.560800 \mathrm{E}-03$ \\
\hline Smoker-vs-Reformed smoker1 & $1.53600000496468 \mathrm{E}-08$ \\
\hline Smoker-vs-Reformed smoker2 & $1.978210 \mathrm{E}-03$ \\
\hline Reformed smoker1-vs-Reformed smoker2 & 1.955780E-04 \\
\hline \multicolumn{2}{|l|}{ Cancer stages } \\
\hline Stage1-vs-Stage2 & $4.427500 \mathrm{E}-02$ \\
\hline \multicolumn{2}{|l|}{ Tissue types } \\
\hline NOS-vs-Mixed & $2.900200 \mathrm{E}-02$ \\
\hline NOS-vs-LBC-Non Mucinous & $2.038200 \mathrm{E}-03$ \\
\hline NOS-vs-Solid Pattern Predominant & $2.599600 \mathrm{E}-02$ \\
\hline NOS-vs-Acinar & 5.378900E-03 \\
\hline NOS-vs-LBC-Mucinous & $1.70860000003881 \mathrm{E}-06$ \\
\hline NOS-vs-Papillary & $1.027880 \mathrm{E}-04$ \\
\hline Mixed-vs-Solid Pattern Predominant & $1.196000 \mathrm{E}-02$ \\
\hline Mixed-vs-LBC-Mucinous & $4.00150000001709 \mathrm{E}-06$ \\
\hline Mixed-vs-Papillary & 4.149500E-02 \\
\hline Clear Cell-vs-LBC-Non Mucinous & $4.028200 \mathrm{E}-02$ \\
\hline Clear Cell-vs-Papillary & $2.063500 \mathrm{E}-02$ \\
\hline LBC-Non Mucinous-vs-Solid Pattern Predominant & 4.452100E-02 \\
\hline LBC-Non Mucinous-vs-LBC-Mucinous & $1.174210 \mathrm{E}-02$ \\
\hline Solid Pattern Predominant-vs-LBC-Mucinous & $1.926450 \mathrm{E}-02$ \\
\hline Solid Pattern Predominant-vs-Mucinous carcinoma & $4.480600 \mathrm{E}-03$ \\
\hline Solid Pattern Predominant-vs-Papillary & $3.801800 \mathrm{E}-02$ \\
\hline Acinar-vs-LBC-Mucinous & 1.693590E-02 \\
\hline LBC-Mucinous-vs-Papillary & $1.804030 \mathrm{E}-02$ \\
\hline \multicolumn{2}{|l|}{ TP53 Mutant } \\
\hline TP53-Mutant-vs-TP53-Non Mutant & $1.81166193158333 \mathrm{E}-12$ \\
\hline
\end{tabular}

Note: Reformed smoker 1, Reformed smoker (<15 years); Reformed smoker 2, Reformed smoker ( $>15$ years); NOS, Lung Adenocarcinoma-Not Otherwise Specified; Mixed, Lung Adenocarcinoma Mixed subtype; Clear Cell, Lung Clear Cell 
Adenocarcinoma; LBC-Nonmucinous, Lung Bronchioloalveolar Carcinoma Non mucinous; Solid Pattern Predominant, Lung Solid Pattern Predominant Adenocarcinoma; Acinar, Lung Acinar Adenocarcinoma; LBC-Mucinous, Lung Bronchioloalveolar Carcinoma Mucinous; Mucinous, Mucinous (Colloid) Carcinoma; Papillary, Lung Papillary Adenocarcinoma; Mucinous, Lung Mucinous Adenocarcinoma; Micropapillary, Lung Micropapillary Adenocarcinoma; Signet Ring, Lung Signet Ring Adenocarcinoma.

Table 2. COX regression analysis of the correlation between clinicopathological features with prognosis.

\begin{tabular}{lcccc}
\hline Clinical characteristics & HR & HR 95\% CI low & HR 95\% CI up & $p$ value \\
\hline a & & & & \\
Age & 1.057406203 & 0.713393673 & 1.567308372 & 0.781014797 \\
Gender & 1.00097432 & 0.698837323 & 1.433737948 & 0.995761563 \\
Stage & 1.64465101 & 1.396688 & 1.93663649 & $2.42 \mathrm{E}-09$ \\
T & 1.623091548 & 1.309819761 & 2.011289072 & $9.57 \mathrm{E}-06$ \\
M & 1.681168333 & 0.923680619 & 3.059853055 & 0.08910352 \\
N & 1.792676516 & 1.464854278 & 2.193862653 & $1.47 \mathrm{E}-08$ \\
MTFR2 & 1.096892408 & 1.002541345 & 1.200123029 & 0.043876089 \\
b & & & & \\
Stage & 1.37421707 & 1.094758223 & 1.725013356 & 0.006135636 \\
T & 1.223211397 & 0.971744105 & 1.539753227 & 0.086187124 \\
N & 1.283860776 & 0.991173421 & 1.662976889 & 0.05838382 \\
MTFR2 & 1.062548364 & 0.964968929 & 1.169995211 & 0.217045634 \\
\hline
\end{tabular}

Table 3. The top 10 genes with positive and negative correlation to MTFR2. 


\begin{tabular}{llll}
\hline Gene & Corgene & Cor & $p$ value \\
\hline MTFR2 & CENPW & 0.876 & $1.08 \mathrm{E}-170$ \\
MTFR2 & TTK & 0.849 & $7.63 \mathrm{E}-150$ \\
MTFR2 & NCAPH & 0.848 & $4.00 \mathrm{E}-149$ \\
MTFR2 & RAD51 & 0.835 & $1.43 \mathrm{E}-140$ \\
MTFR2 & CENPA & 0.827 & $2.59 \mathrm{E}-135$ \\
MTFR2 & KIF2C & 0.817 & $2.83 \mathrm{E}-129$ \\
MTFR2 & BUB1 & 0.817 & $1.98 \mathrm{E}-129$ \\
MTFR2 & CDCA8 & 0.816 & $5.28 \mathrm{E}-129$ \\
MTFR2 & HJURP & 0.814 & $7.81 \mathrm{E}-128$ \\
MTFR2 & CDCA5 & 0.814 & $1.26 \mathrm{E}-127$ \\
MTFR2 & C16orf89 & -0.655 & $7.35 \mathrm{E}-67$ \\
MTFR2 & CRY2 & -0.628 & $3.92 \mathrm{E}-60$ \\
MTFR2 & C1orf116 & -0.585 & $1.60 \mathrm{E}-50$ \\
MTFR2 & CYP4B1 & -0.583 & $5.72 \mathrm{E}-50$ \\
MTFR2 & SELENBP1 & -0.58 & $2.16 \mathrm{E}-49$ \\
MTFR2 & CACNA2D2 & -0.576 & $1.56 \mathrm{E}-48$ \\
MTFR2 & ADGRF5 & -0.568 & $4.68 \mathrm{E}-47$ \\
MTFR2 & NAPSA & -0.561 & $1.09 \mathrm{E}-45$ \\
MTFR2 & SNED1 & -0.558 & $4.89 \mathrm{E}-45$ \\
MTFR2 & NR3C2 & -0.557 & $6.44 \mathrm{E}-45$ \\
\hline
\end{tabular}

Note: Corgene, co-expressed gene; Cor, correlation coefficient.

Table 4. MTFR2 co-expressed genes by KEGG. 


\begin{tabular}{llccc}
\hline ID & Description & Count & $p$ value & $p$ adjust \\
\hline hsa04110 & Cell cycle & 49 & $2.54 \mathrm{E}-26$ & $7.53 \mathrm{E}-24$ \\
hsa03030 & DNA replication & 26 & $1.92 \mathrm{E}-23$ & $2.84 \mathrm{E}-21$ \\
hsa03050 & Proteasome & 19 & $2.45 \mathrm{E}-11$ & $2.42 \mathrm{E}-09$ \\
hsa03440 & Homologous recombination & 17 & $2.60 \mathrm{E}-10$ & $1.59 \mathrm{E}-08$ \\
hsa03430 & Mismatch repair & 13 & $2.69 \mathrm{E}-10$ & $1.59 \mathrm{E}-08$ \\
hsa03013 & RNA transport & 35 & $5.88 \mathrm{E}-09$ & $2.90 \mathrm{E}-07$ \\
hsa03460 & Fanconi anemia pathway & 17 & $3.58 \mathrm{E}-08$ & $1.52 \mathrm{E}-06$ \\
hsa03040 & Spliceosome & 28 & $4.24 \mathrm{E}-07$ & $1.57 \mathrm{E}-05$ \\
hsa03410 & Base excision repair & 12 & $6.52 \mathrm{E}-07$ & $2.14 \mathrm{E}-05$ \\
hsa03420 & Nucleotide excision repair & 14 & $1.25 \mathrm{E}-06$ & $3.69 \mathrm{E}-05$ \\
hsa04114 & Oocyte meiosis & 24 & $2.97 \mathrm{E}-06$ & $8.00 \mathrm{E}-05$ \\
hsa00670 & One carbon pool by folate & 8 & $2.25 \mathrm{E}-05$ & 0.000555487 \\
hsa03008 & Ribosome biogenesis in eukaryotes & 20 & $3.63 \mathrm{E}-05$ & 0.000826201 \\
hsa04218 & Cellular senescence & 24 & 0.000136465 & 0.002885268 \\
hsa04914 & Progesterone-mediated oocyte maturation & 17 & 0.000252864 & 0.004989844 \\
hsa00240 & Pyrimidine metabolism & 12 & 0.000297856 & 0.00551034 \\
hsa05012 & Parkinson disease & 31 & 0.000505075 & 0.008794241 \\
hsa05166 & Human T-cell leukemia virus 1 infection & 28 & 0.000610833 & 0.010044803 \\
hsa00270 & Cysteine and methionine metabolism & 10 & 0.001213762 & 0.018909138 \\
hsa04115 & p53 signaling pathway & 12 & 0.002598093 & 0.038451783 \\
\hline & & & &
\end{tabular}

Table 5. GSEA displayed the main signal pathways derived from high expression of MTFR2 enrichment. 


\begin{tabular}{|c|c|c|c|}
\hline Name & Size & Nes & Nom $p$ value \\
\hline KEGG_CELL_CYCLE & 124 & 2.5325148 & 0 \\
\hline KEGG_SPLICEOSOME & 126 & 2.342574 & 0 \\
\hline KEGG_OOCYTE_MEIOSIS & 112 & 2.3013418 & 0 \\
\hline KEGG_MISMATCH_REPAIR & 23 & 2.2582684 & 0 \\
\hline KEGG_DNA_REPLICATION & 36 & 2.210251 & 0 \\
\hline KEGG_HOMOLOGOUS_RECOMBINATION & 28 & 2.207216 & 0 \\
\hline KEGG_RNA_DEGRADATION & 57 & 2.2033587 & 0 \\
\hline KEGG_PROGESTERONE_MEDIATED_ & 85 & 2.1570013 & 0 \\
\hline \multicolumn{4}{|l|}{ OOCYTE_MATURATION } \\
\hline KEGG_PROTEASOME & 44 & 2.108183 & 0 \\
\hline KEGG_BASE_EXCISION_REPAIR & 33 & 2.097559 & 0 \\
\hline KEGG_PATHOGENIC_ESCHERICHIA_COLI_INFECTION & 55 & 2.096588 & 0 \\
\hline KEGG_GLYOXYLATE_AND_ & 16 & 2.0935147 & 0 \\
\hline \multicolumn{4}{|l|}{ DICARBOXYLATE_METABOLISM } \\
\hline KEGG_BASAL_TRANSCRIPTION_FACTORS & 35 & 2.051244 & 0 \\
\hline KEGG_PYRIMIDINE_METABOLISM & 98 & 2.0281448 & 0 \\
\hline KEGG_ONE_CARBON_POOL_BY_FOLATE & 17 & 2.0059762 & 0 \\
\hline KEGG_P53_SIGNALING_PATHWAY & 68 & 1.96642 & 0 \\
\hline KEGG_PURINE_METABOLISM & 157 & 1.9532791 & 0 \\
\hline KEGG_NUCLEOTIDE_EXCISION_REPAIR & 44 & 2.0500638 & 0.001831502 \\
\hline KEGG_UBIQUITIN_MEDIATED_PROTEOLYSIS & 133 & 1.7833992 & 0.003868472 \\
\hline KEGG_CYSTEINE_AND_METHIONINE_METABOLISM & 34 & 1.8469852 & 0.003883495 \\
\hline KEGG_FRUCTOSE_AND_MANNOSE_METABOLISM & 33 & 1.821762 & 0.011516315 \\
\hline KEGG_PENTOSE_PHOSPHATE_PATHWAY & 27 & 1.7594053 & 0.011650485 \\
\hline KEGG_N_GLYCAN_BIOSYNTHESIS & 46 & 1.6938915 & 0.023346303 \\
\hline KEGG_BLADDER_CANCER & 42 & 1.5152026 & 0.0251938 \\
\hline KEGG_RIBOFLAVIN_METABOLISM & 16 & 1.5648233 & 0.027675277 \\
\hline KEGG_PANCREATIC_CANCER & 70 & 1.4912151 & 0.03131524 \\
\hline KEGG_GLYCOLYSIS_GLUCONEOGENESIS & 62 & 1.5601822 & 0.036398467 \\
\hline KEGG_HUNTINGTONS_DISEASE & 177 & 1.6180334 & 0.046511628 \\
\hline KEGG_RNA_POLYMERASE & 29 & 1.5426548 & 0.048732944 \\
\hline
\end{tabular}

Table 6. The top 10 Hub genes in PPI Network. 


\begin{tabular}{llc}
\hline Name & Description & Score \\
\hline CDK1 & Cyclin dependent kinase 1 & 192 \\
CDC20 & Cell division cycle 20 & 160 \\
CCNB1 & Cyclin B1 & 138 \\
PLK1 & Polo like kinase 1 & 134 \\
CCNA2 & Cyclin A2 & 129 \\
AURKB & Aurora kinase B & 127 \\
CCNB2 & Cyclin B2 & 124 \\
BUB1B & BUB1 mitotic checkpoint serine/threonine kinase B & 118 \\
MAD2L1 & Mitotic arrest deficient 2 like 1 & 117 \\
BUB1 & BUB1 mitotic checkpoint serine/threonine kinase & 105 \\
\hline
\end{tabular}

Figures
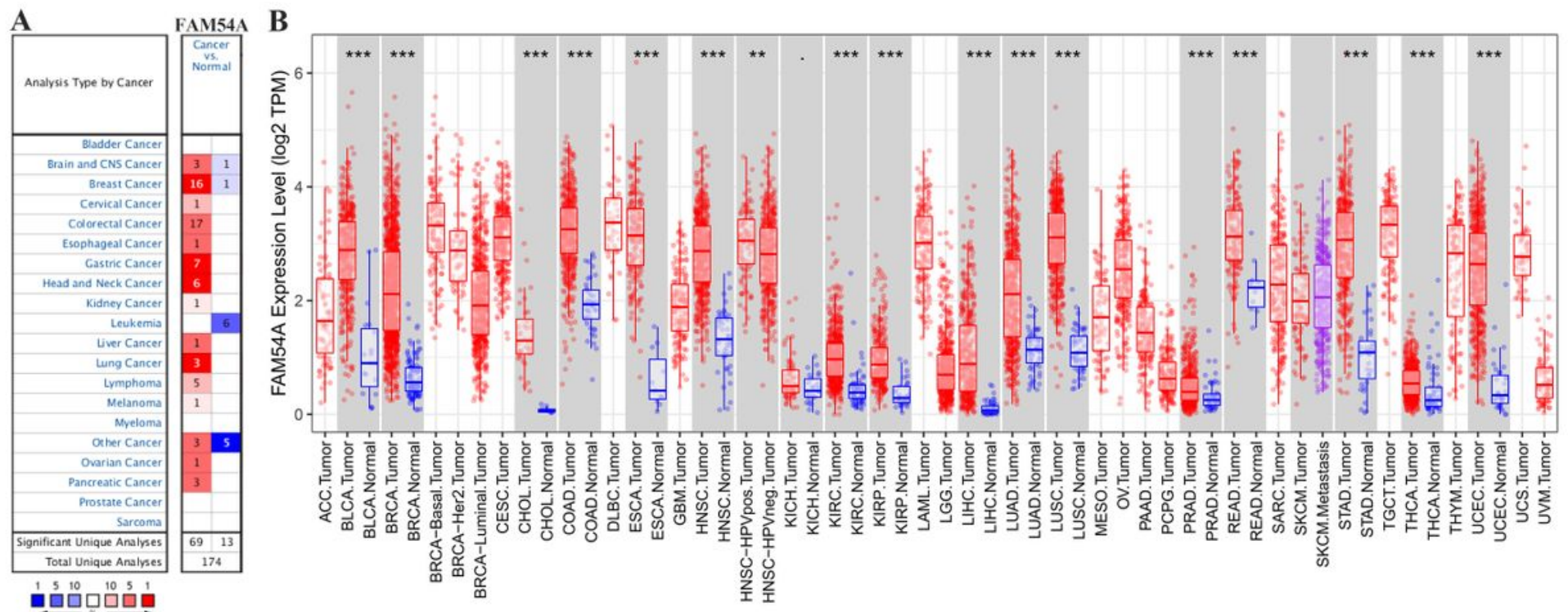

Figure 1

MTFR2 expression in cancer tissues. Note: (A) Oncomine database; (B) Timer database. Note: (A) Red, increased expression of MTFR2 in cancer tissues, blue, decreased expression of MTFR2 in cancer tissues; (B) red, cancer tissues, blue, normal tissues. *, $p<0.05 ; * \star, p<0.01 ; * \star \star, p<0.001$. 

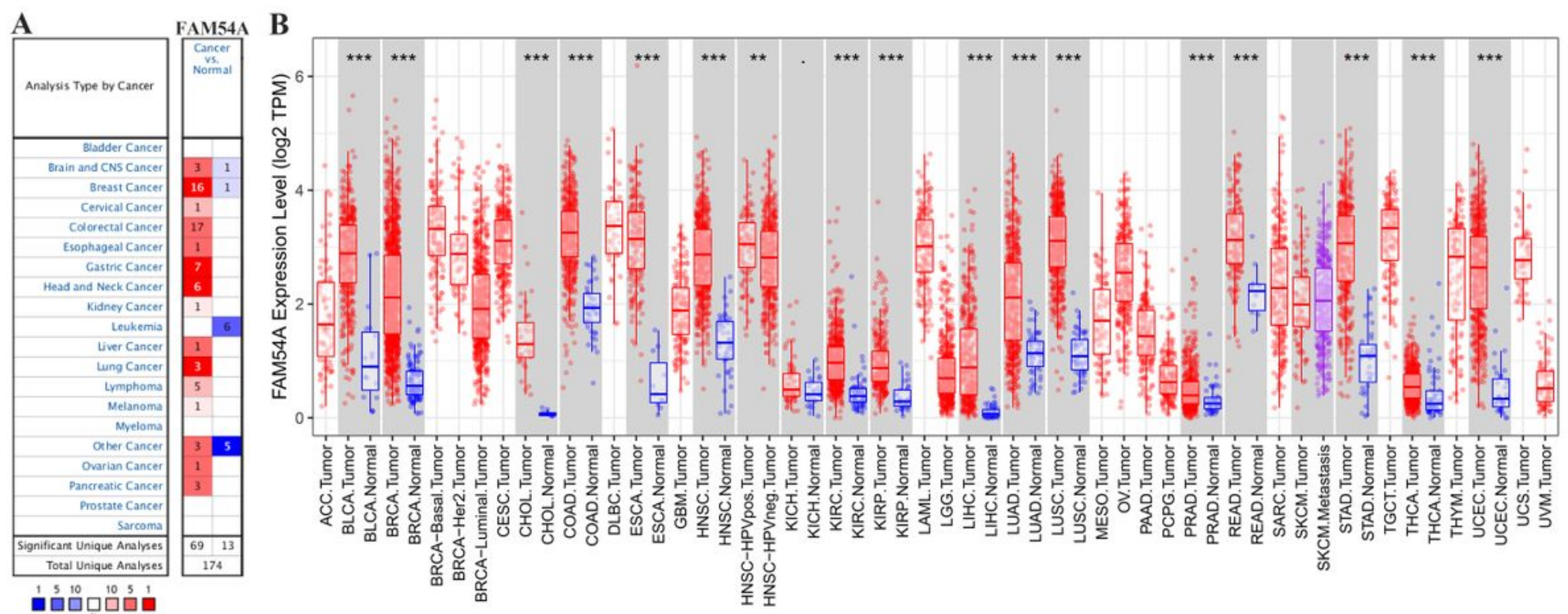

\section{Figure 1}

MTFR2 expression in cancer tissues. Note: (A) Oncomine database; (B) Timer database. Note: (A) Red, increased expression of MTFR2 in cancer tissues, blue, decreased expression of MTFR2 in cancer tissues; (B) red, cancer tissues, blue, normal tissues. *, $p<0.05 ; * \star, p<0.01 ; * \star *, p<0.001$.

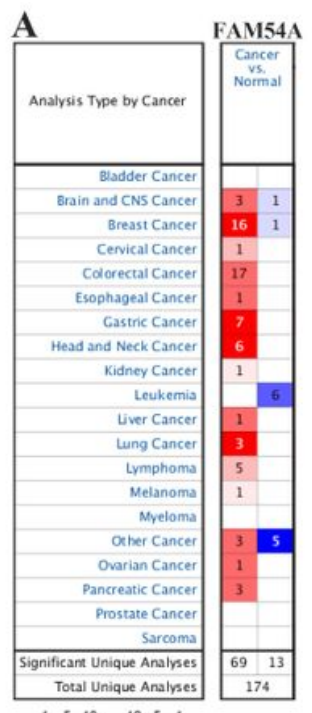

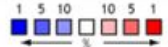

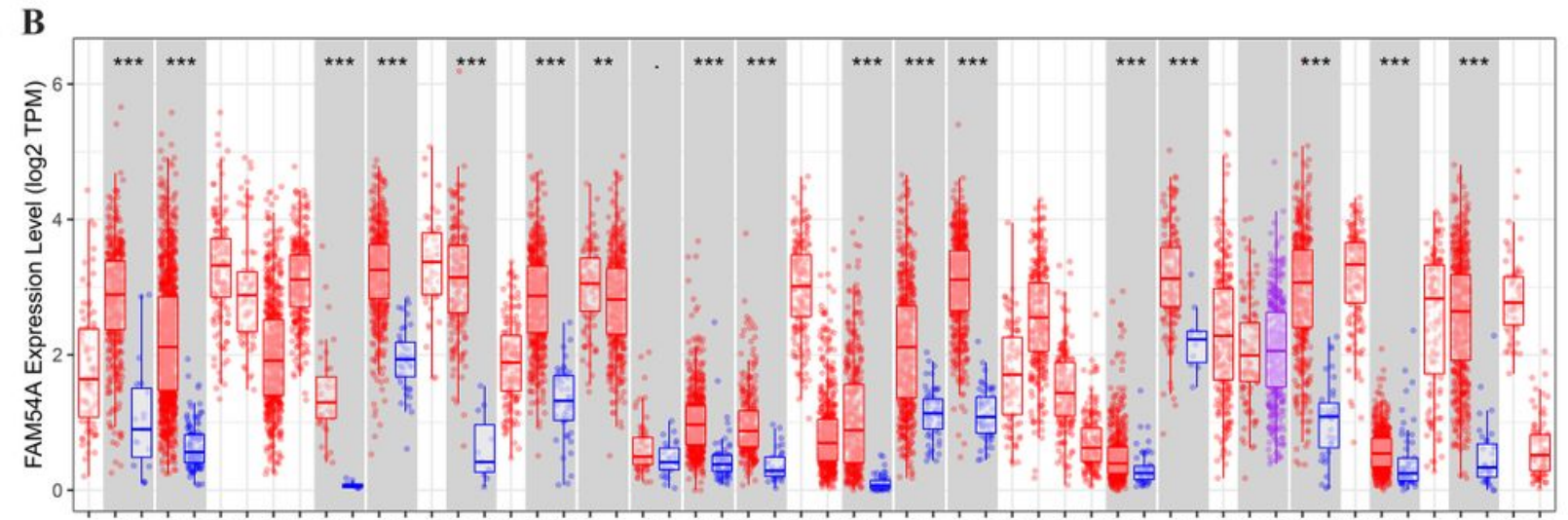

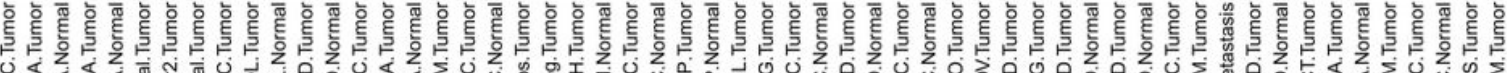

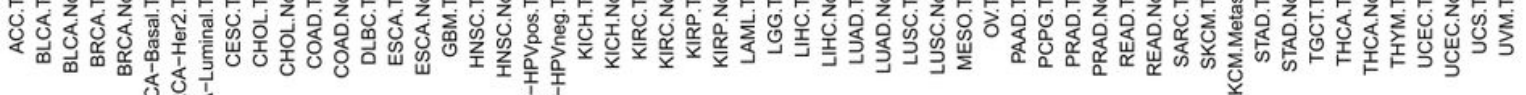

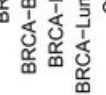

\section{Figure 1}

MTFR2 expression in cancer tissues. Note: (A) Oncomine database; (B) Timer database. Note: (A) Red, increased expression of MTFR2 in cancer tissues, blue, decreased expression of MTFR2 in cancer tissues; (B) red, cancer tissues, blue, normal tissues. *, $p<0.05 ; * \star, p<0.01$; ${ }^{* \star *}, p<0.001$. 

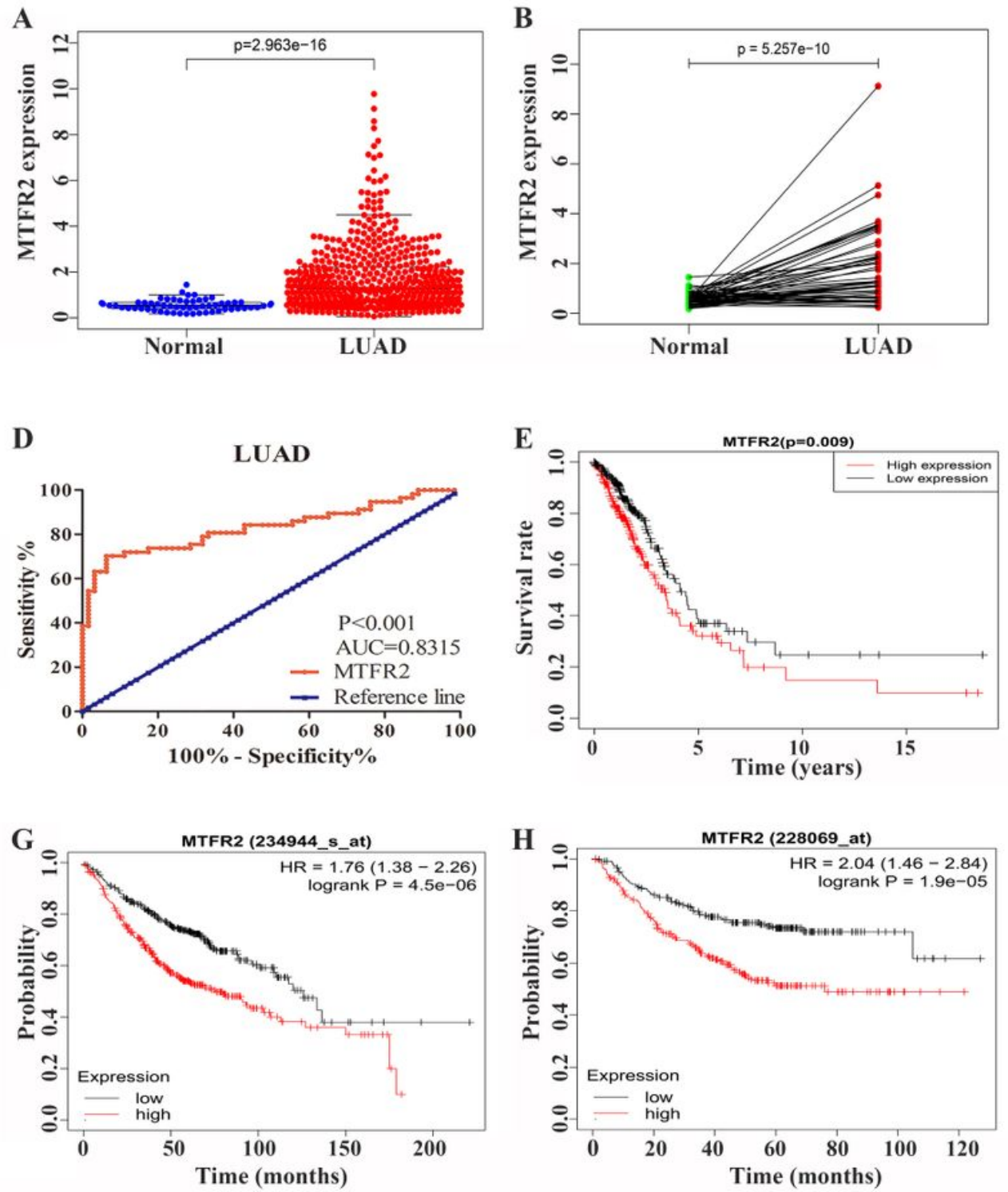

C
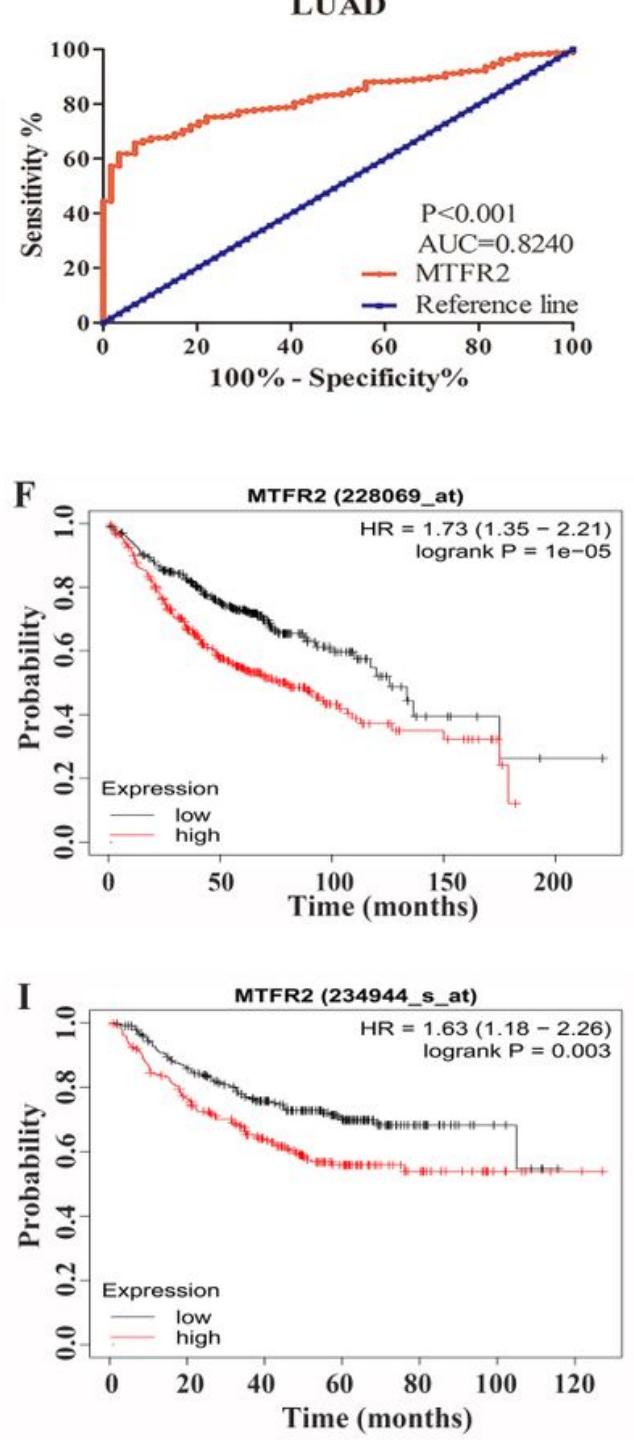

\section{Figure 2}

The expression of MTFR2 was correlated to the diagnosis and prognosis in LUAD patients. Note: (A) Expression of MTFR2 in unpaired LUAD tissues; (B) Expression of MTFR2 in 57 paired LUAD tissues; (C) The diagnostic value of MTFR2 in unpaired LUAD tissues; (D)The diagnostic value of MTFR2 in 57 paired LUAD tissues; (E) The expression of MTFR2 in TCGA database was correlated with the overall survival of LUAD patients; (F-G) The levels of MTFR2 (228069_at) and MTFR2 (234944_at) were correlated to the overall survival of LUAD patients in Kaplan-Meier Plotter database; $(\mathrm{H}-\mathrm{I})$ The levels of MTFR2 (228069_at) and MTFR2 (234944_at) were correlated to the early progression in LUAD patients in the Kaplan-Meier Plotter database. Note: Normal, normal lung tissue; LUAD, lung adenocarcinoma tissue; AUC, Area under curve. 
A

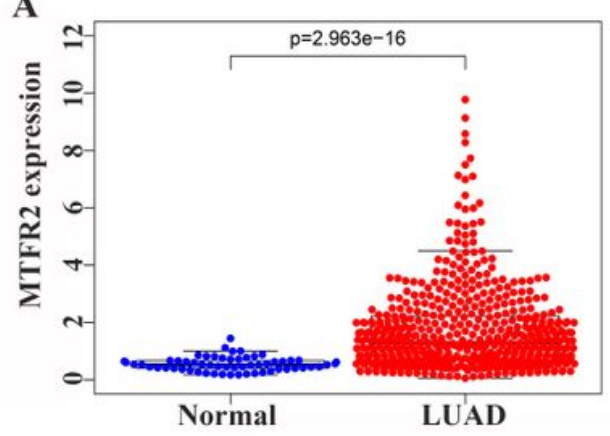

D
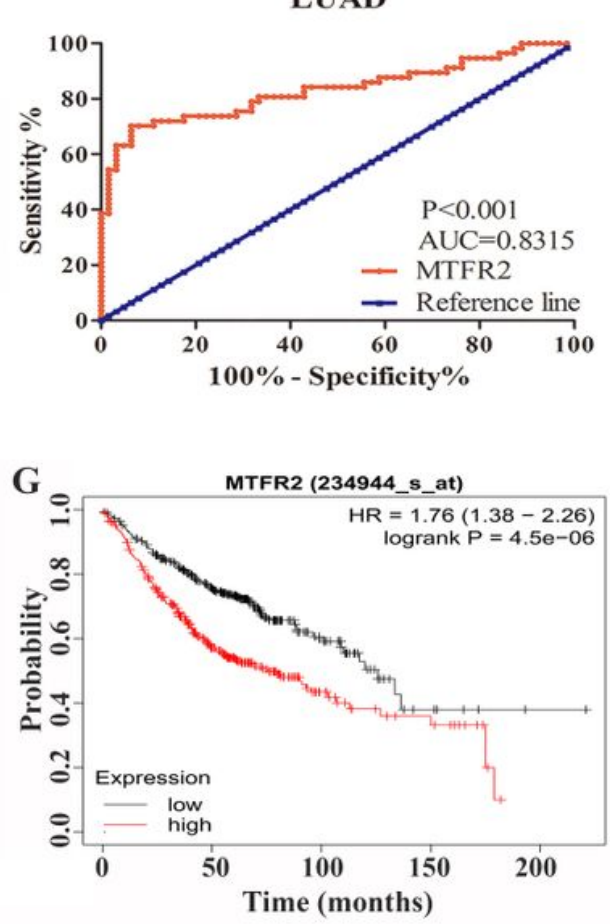

B

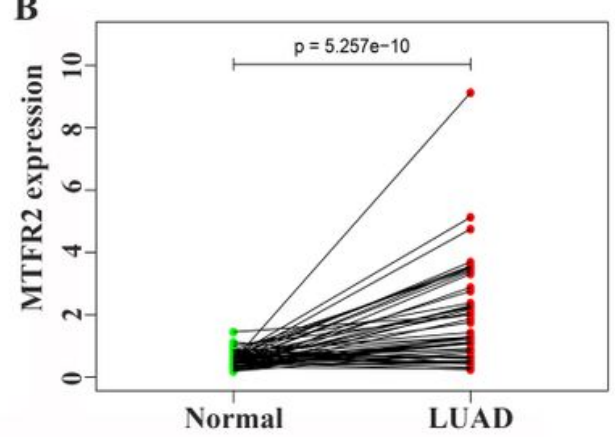

E
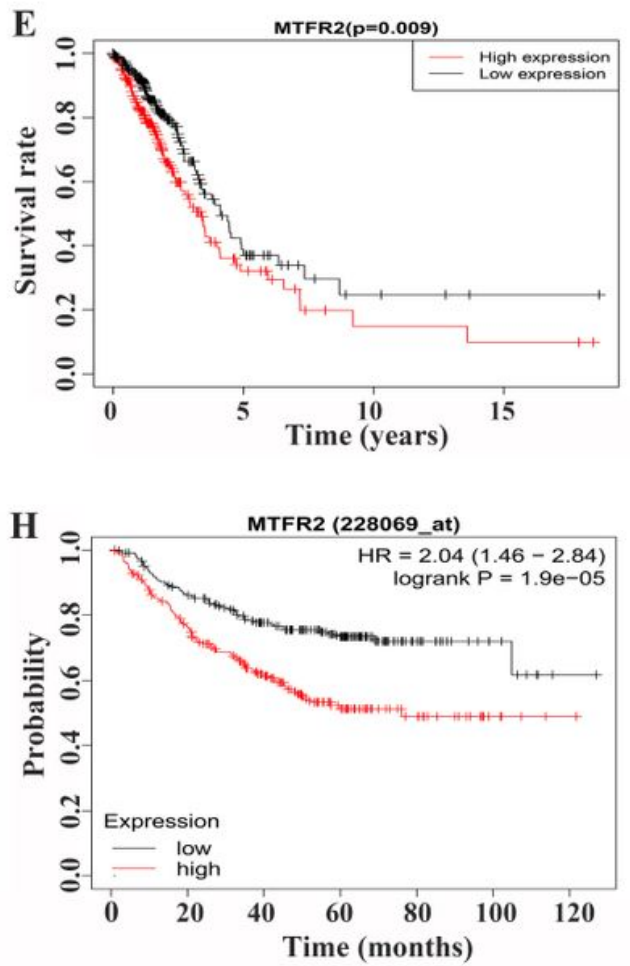

C
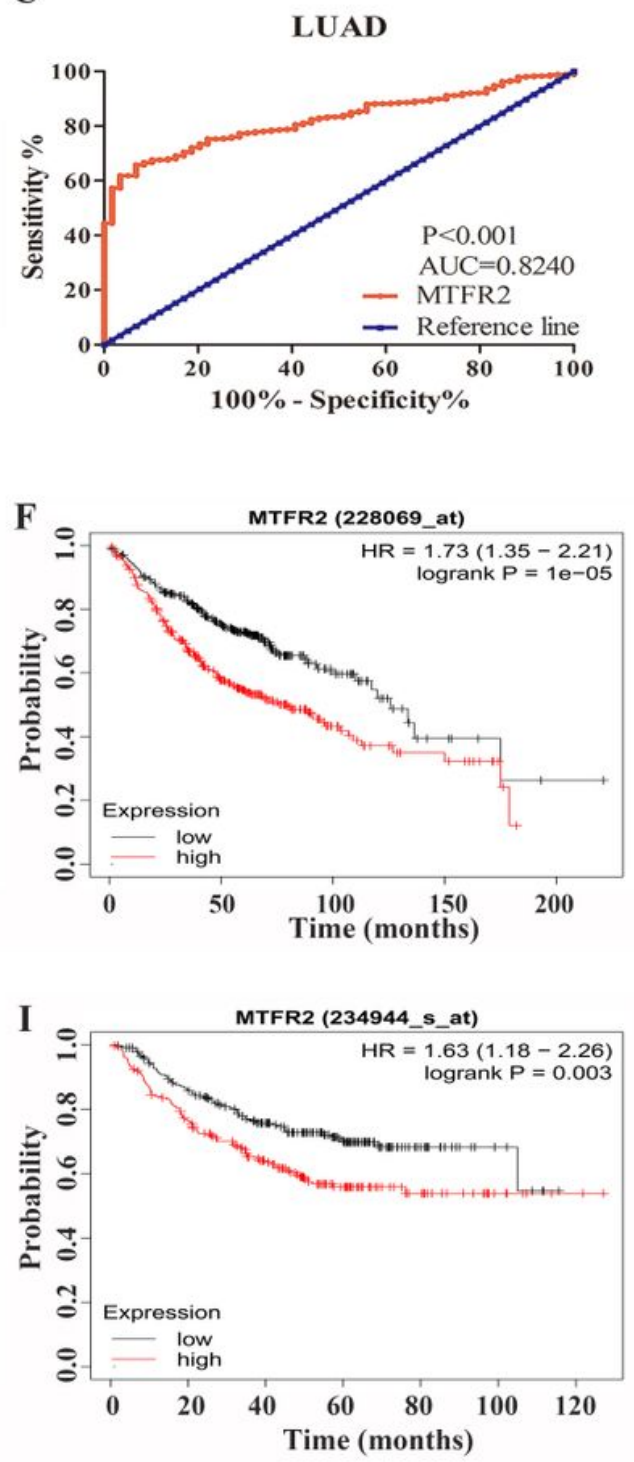

Figure 2

The expression of MTFR2 was correlated to the diagnosis and prognosis in LUAD patients. Note: (A) Expression of MTFR2 in unpaired LUAD tissues; (B) Expression of MTFR2 in 57 paired LUAD tissues; (C) The diagnostic value of MTFR2 in unpaired LUAD tissues; (D)The diagnostic value of MTFR2 in 57 paired LUAD tissues; (E) The expression of MTFR2 in TCGA database was correlated with the overall survival of LUAD patients; (F-G) The levels of MTFR2 (228069_at) and MTFR2 (234944_at) were correlated to the overall survival of LUAD patients in Kaplan-Meier Plotter database; (H-I) The levels of MTFR2 (228069_at) and MTFR2 (234944_at) were correlated to the early progression in LUAD patients in the Kaplan-Meier Plotter database. Note: Normal, normal lung tissue; LUAD, lung adenocarcinoma tissue; AUC, Area under curve. 

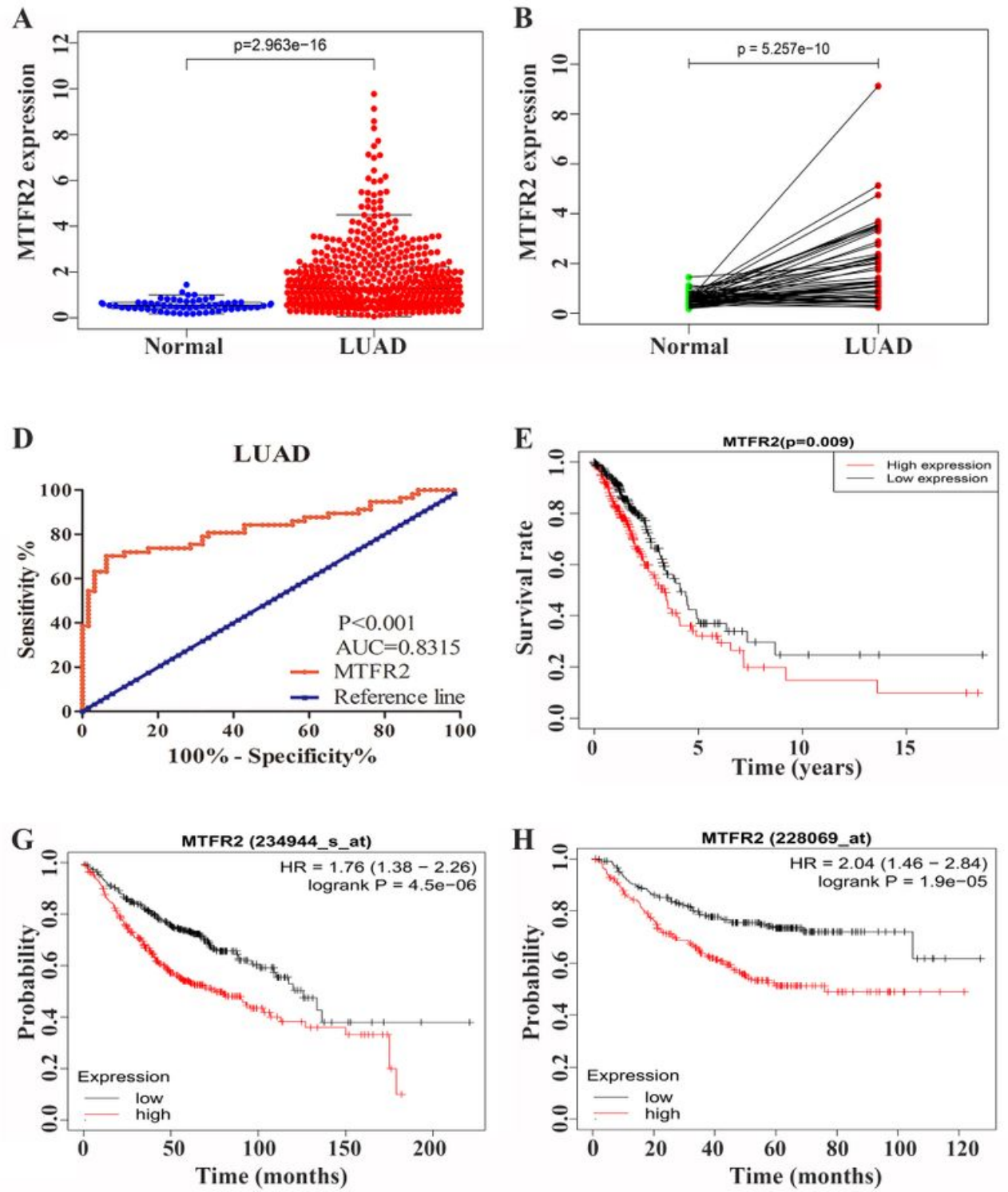

C
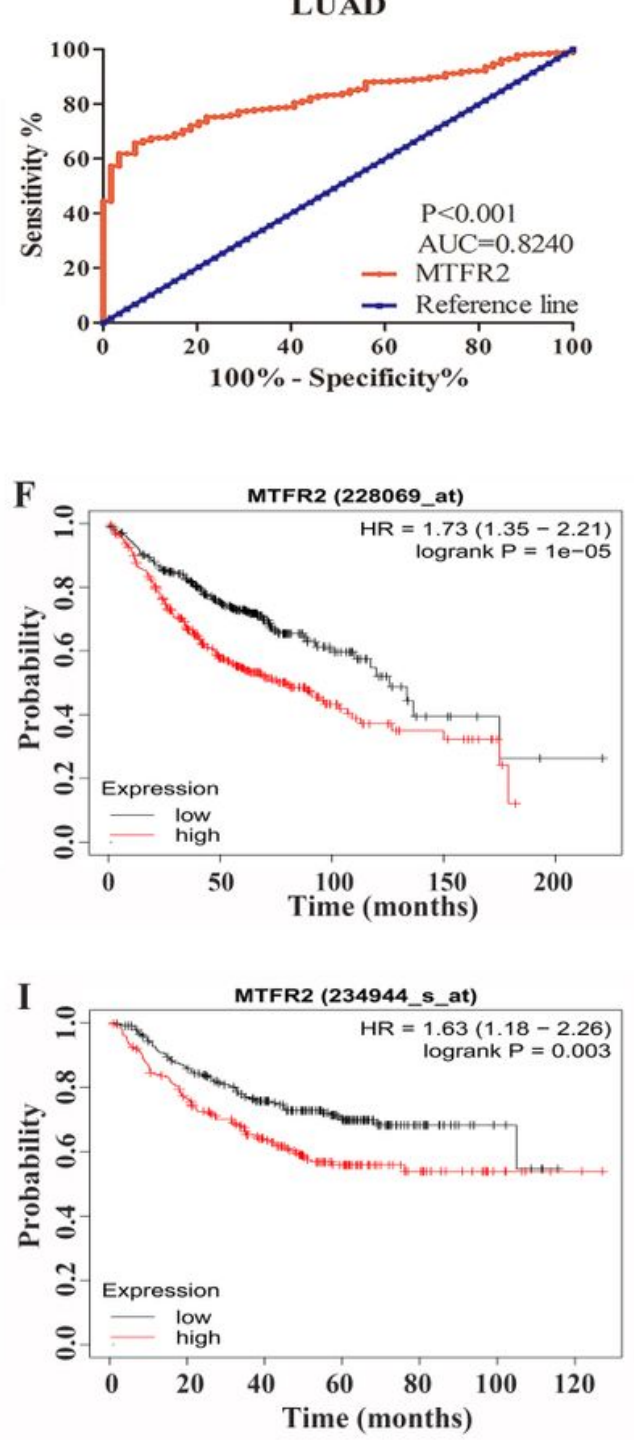

\section{Figure 2}

The expression of MTFR2 was correlated to the diagnosis and prognosis in LUAD patients. Note: (A) Expression of MTFR2 in unpaired LUAD tissues; (B) Expression of MTFR2 in 57 paired LUAD tissues; (C) The diagnostic value of MTFR2 in unpaired LUAD tissues; (D)The diagnostic value of MTFR2 in 57 paired LUAD tissues; (E) The expression of MTFR2 in TCGA database was correlated with the overall survival of LUAD patients; (F-G) The levels of MTFR2 (228069_at) and MTFR2 (234944_at) were correlated to the overall survival of LUAD patients in Kaplan-Meier Plotter database; $(\mathrm{H}-\mathrm{I})$ The levels of MTFR2 (228069_at) and MTFR2 (234944_at) were correlated to the early progression in LUAD patients in the Kaplan-Meier Plotter database. Note: Normal, normal lung tissue; LUAD, lung adenocarcinoma tissue; AUC, Area under curve. 


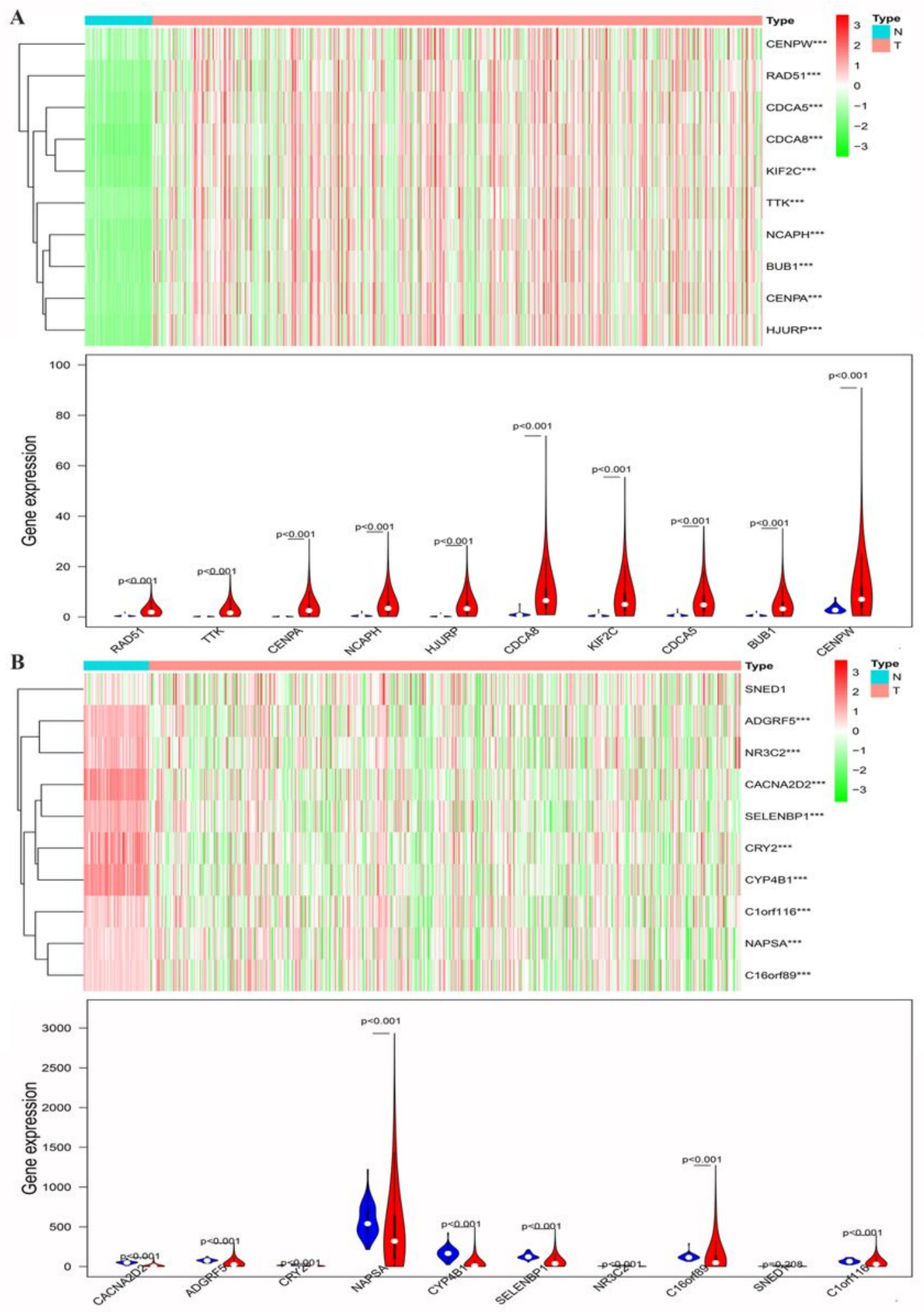

\section{Figure 3}

The expressions of the top 10 positive and negative related genes of MTFR2 in LUAD tissues. Note $(A)$ The top 10 positively correlated genes; (B) The top 10 negatively correlated genes. Note: $\star \star \star, ~ p<0.001 ; N$, normal lung tissue; $\mathrm{T}$, lung adenocarcinoma tissue. 


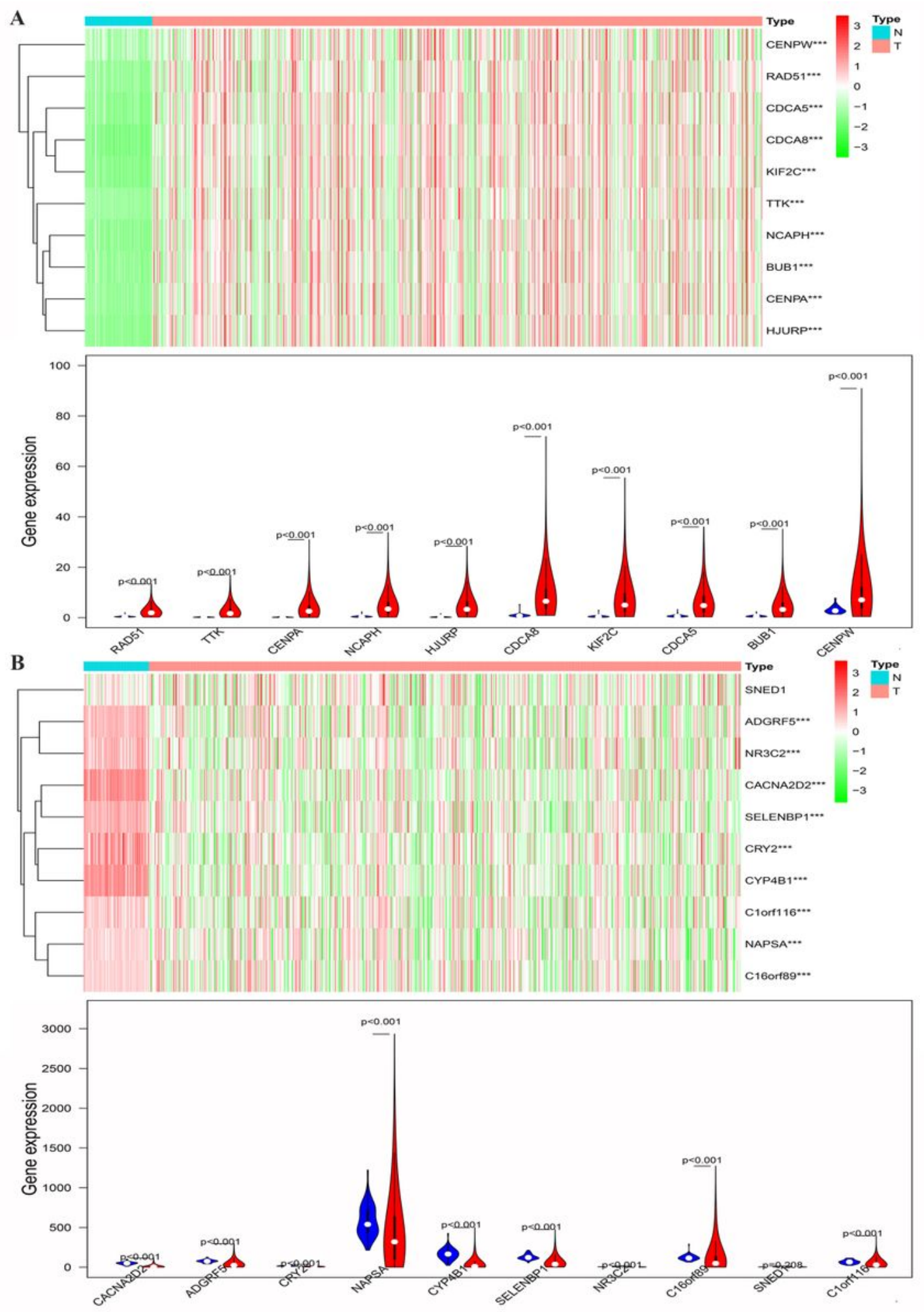

\section{Figure 3}

The expressions of the top 10 positive and negative related genes of MTFR2 in LUAD tissues. Note $(A)$ The top 10 positively correlated genes; (B) The top 10 negatively correlated genes. Note: $\star \star \star, ~ p<0.001 ; N$, normal lung tissue; $\mathrm{T}$, lung adenocarcinoma tissue. 


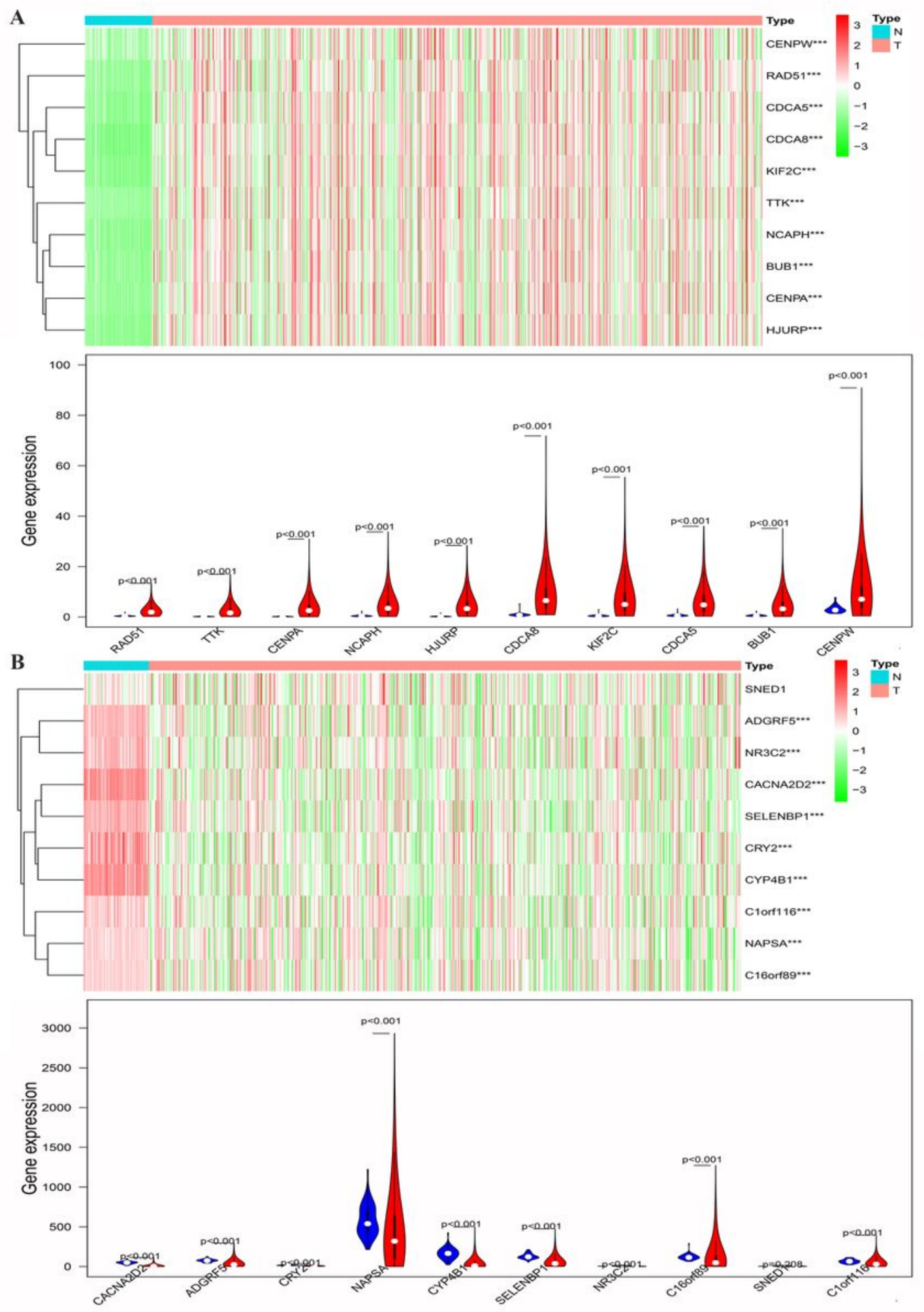

\section{Figure 3}

The expressions of the top 10 positive and negative related genes of MTFR2 in LUAD tissues. Note $(A)$ The top 10 positively correlated genes; (B) The top 10 negatively correlated genes. Note: $\star \star \star, ~ p<0.001 ; N$, normal lung tissue; $\mathrm{T}$, lung adenocarcinoma tissue. 
A

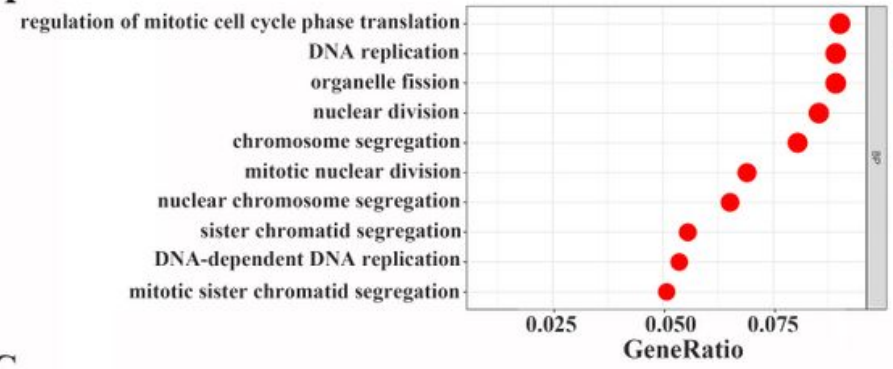

C

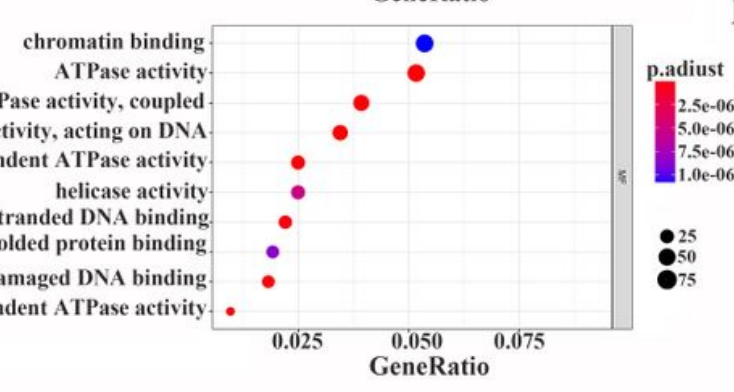

B

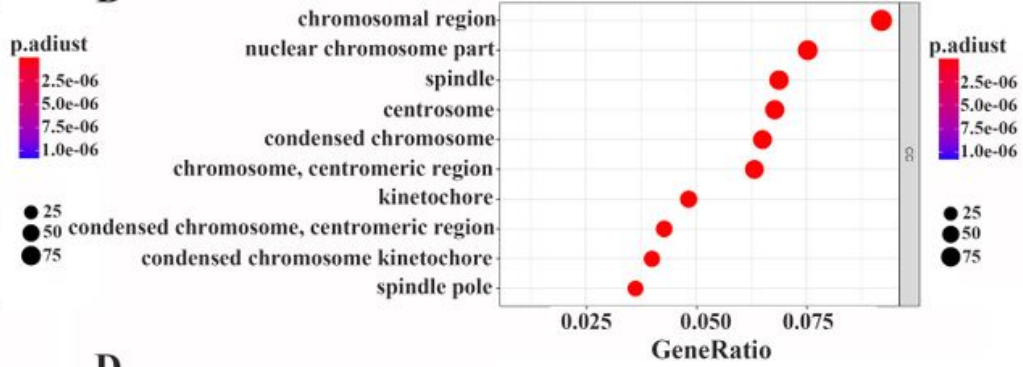

D

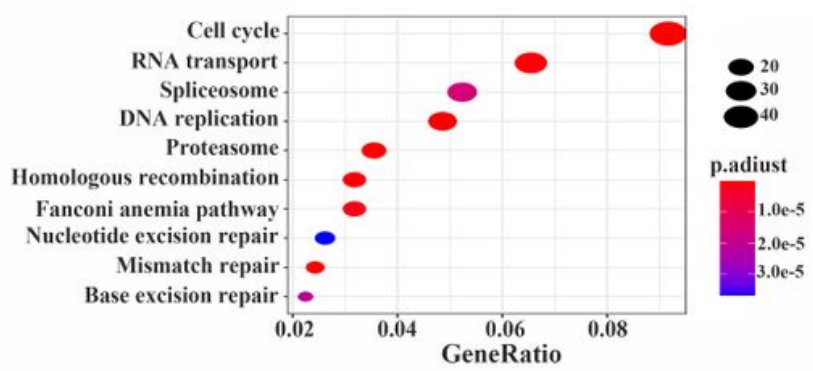

Figure 4

Analysis of MTFR2 co-expressed genes by GO and KEGG. Note囚 (A) Biological processes (BP); (B) Cellular component (CC); (C) Molecular function (MF); (D) KEGG.

A

regulation of mitotic cell cycle phase translation.

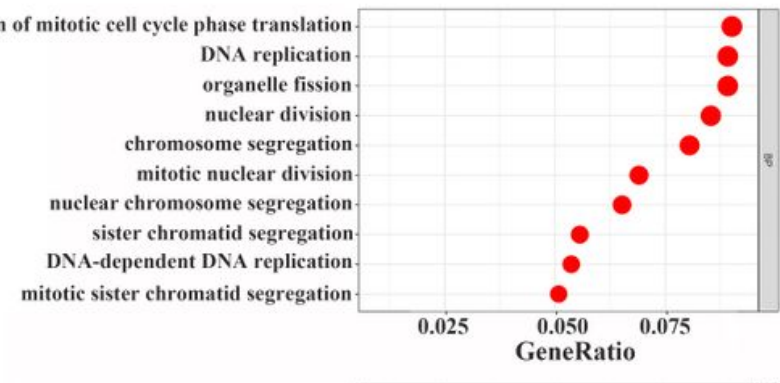

C

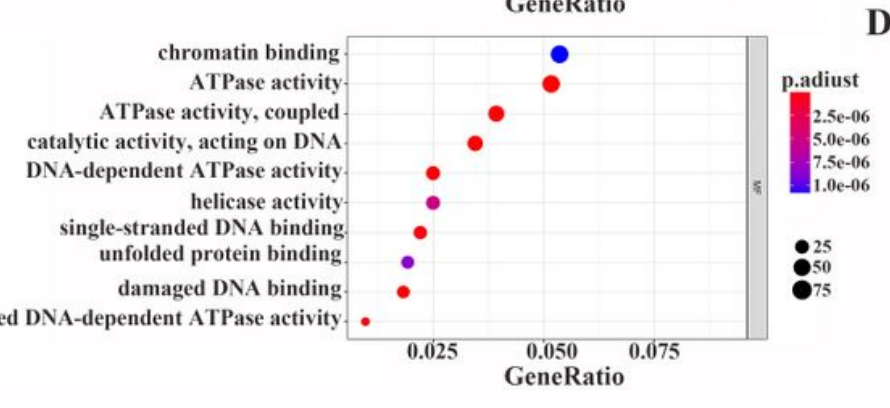

B
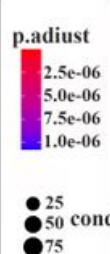

D

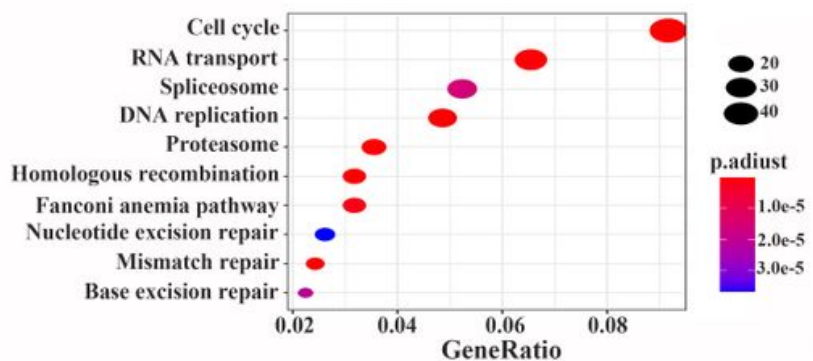

\section{Figure 4}

Analysis of MTFR2 co-expressed genes by GO and KEGG. Note『 (A) Biological processes (BP); (B) Cellular component (CC); (C) Molecular function (MF); (D) KEGG. 
A

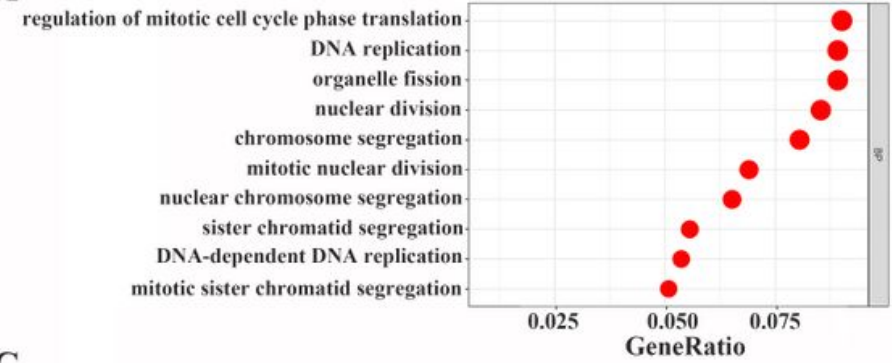

C

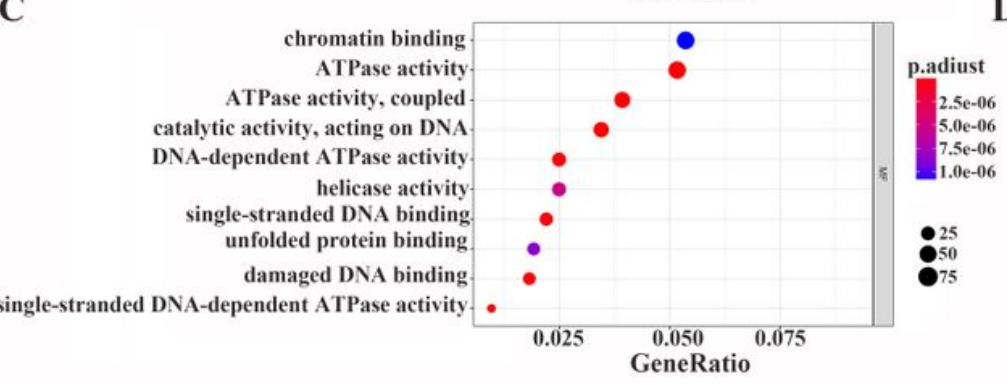

B

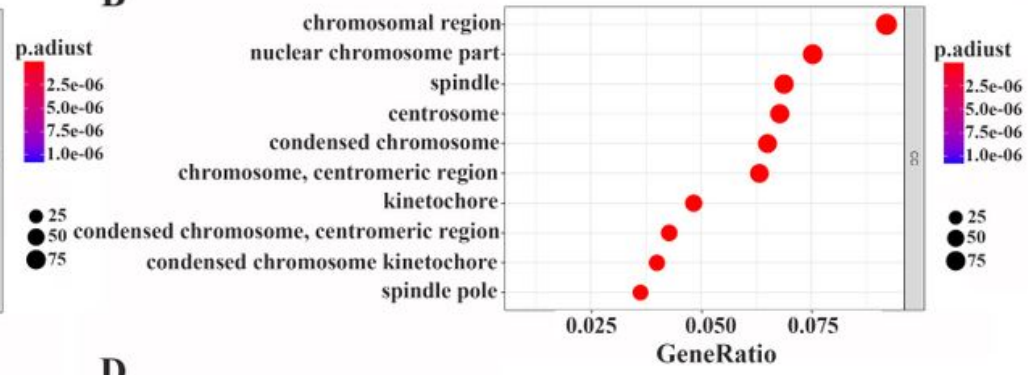

D

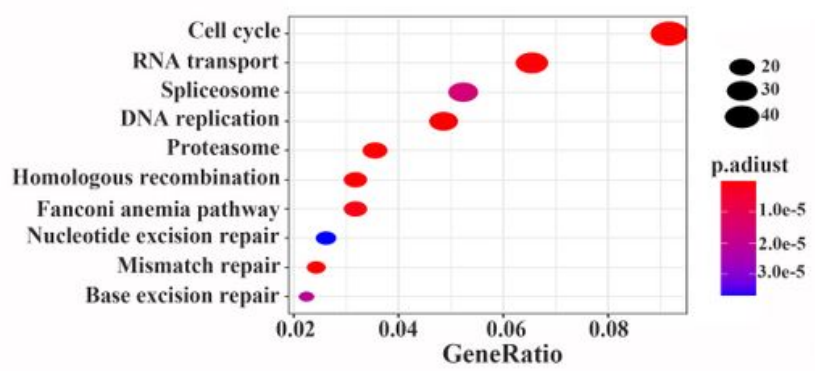

Figure 4

Analysis of MTFR2 co-expressed genes by GO and KEGG. Note囚 (A) Biological processes (BP); (B) Cellular component (CC); (C) Molecular function (MF); (D) KEGG. 
A

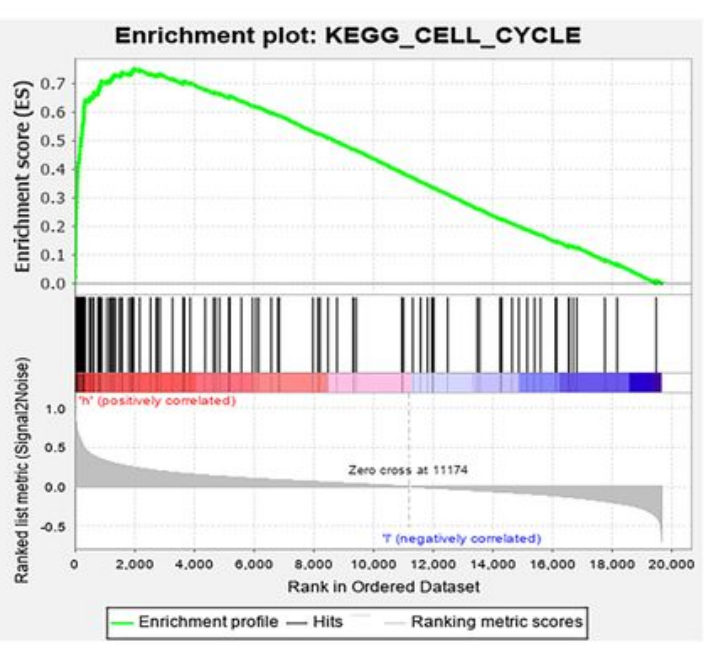

C

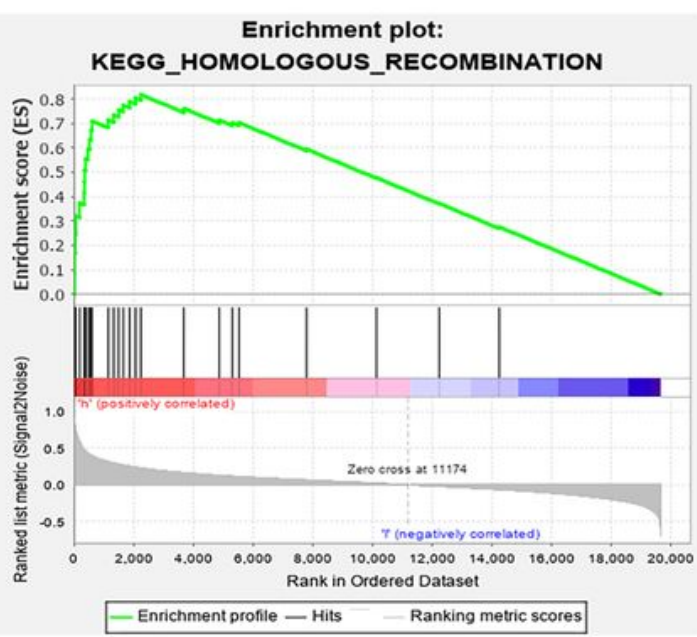

$\mathbf{E}$

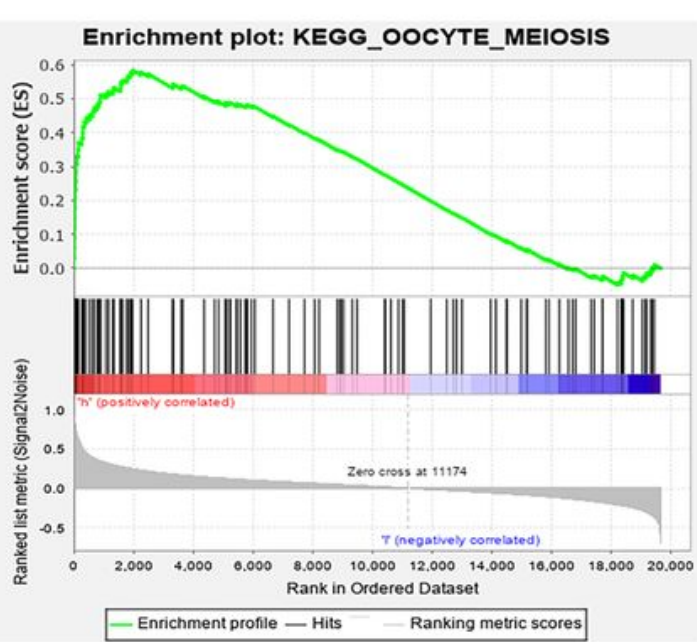

B

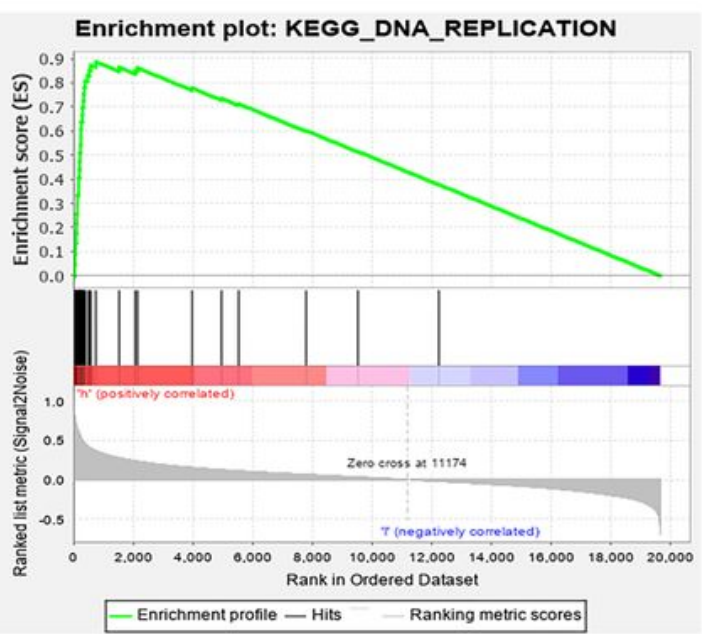

D

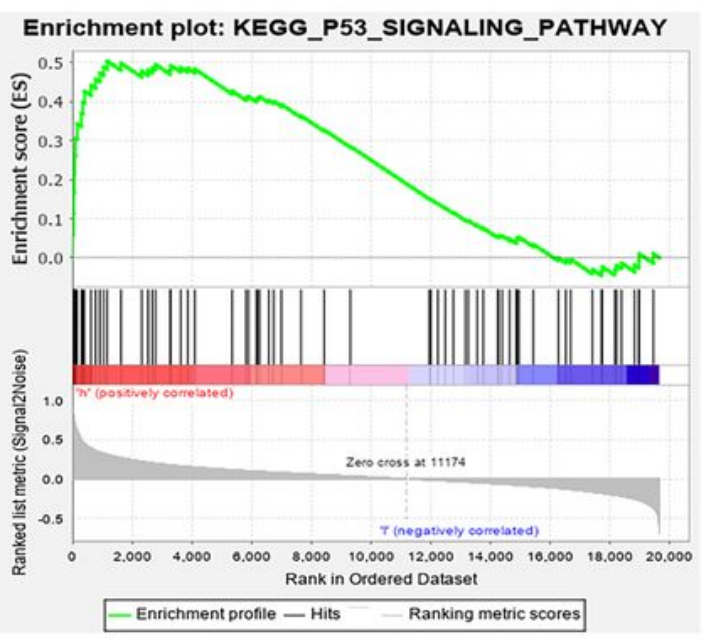

F

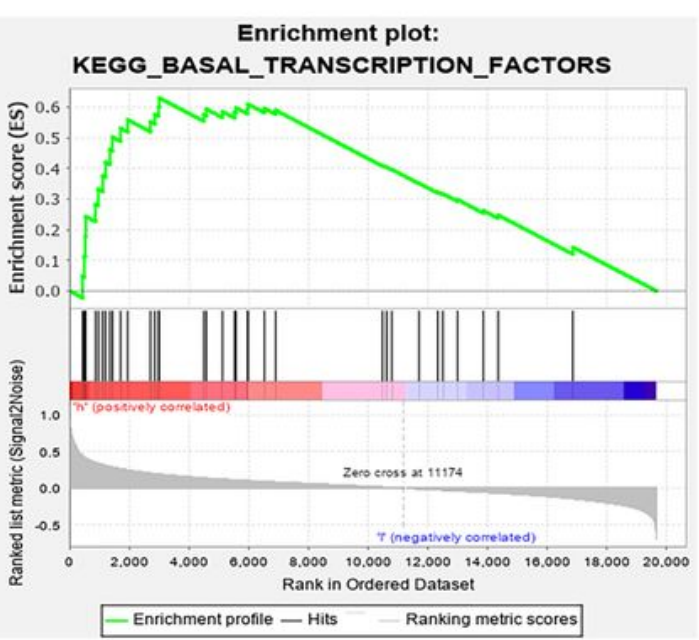

Figure 5

GSEA displayed the main signal pathways derived from high expression of MTFR2 enrichment. Note区 (A) Cell cycle; (B) DNA replication; (C) Homologous recombination; (D) p53 signaling pathway; (E) Oocyte meiosis; (F) Base_excision_repair. 
A

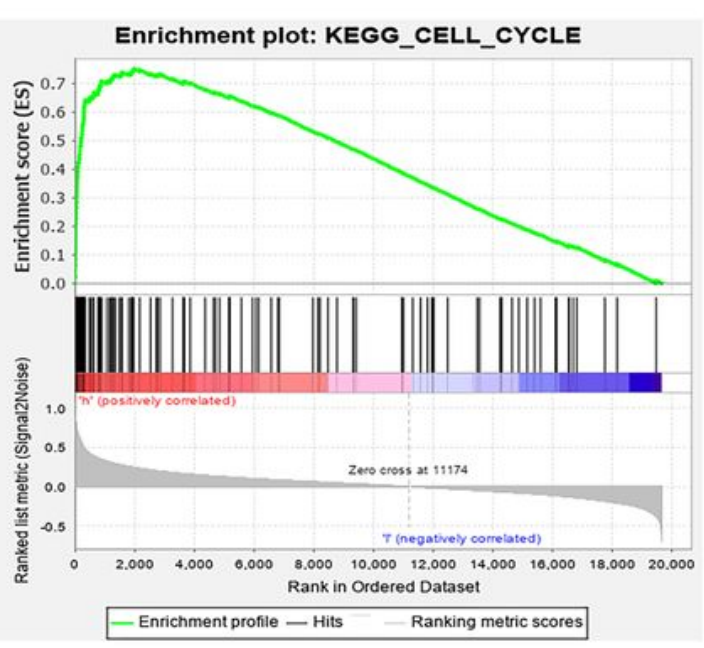

C

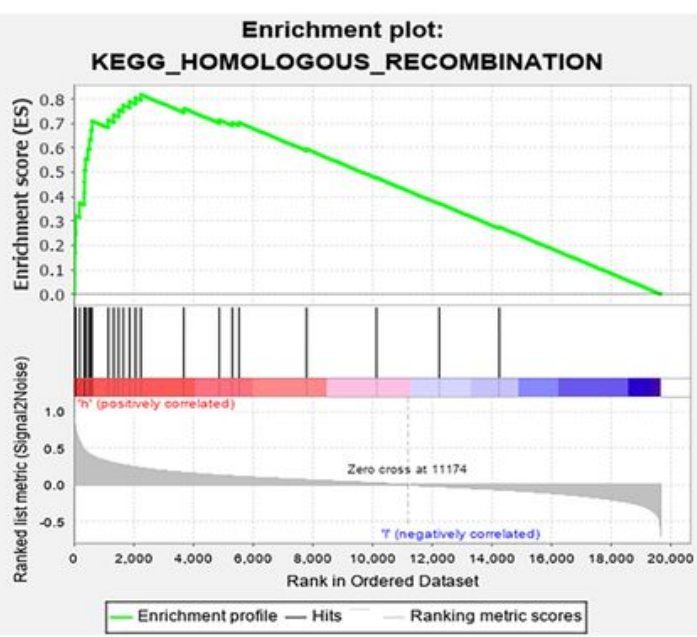

$\mathbf{E}$

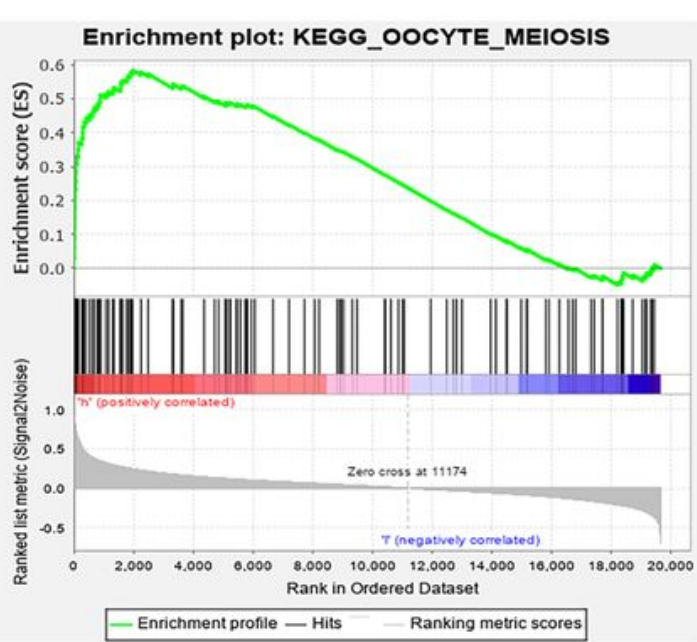

B

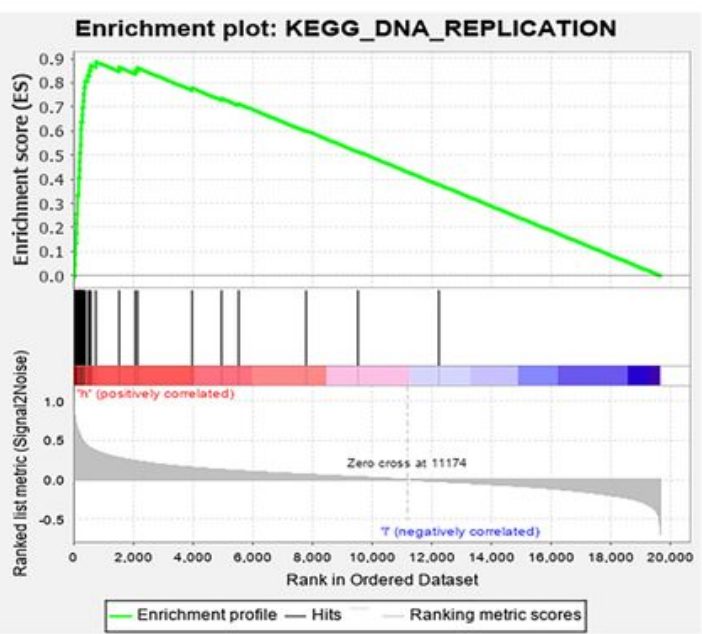

D

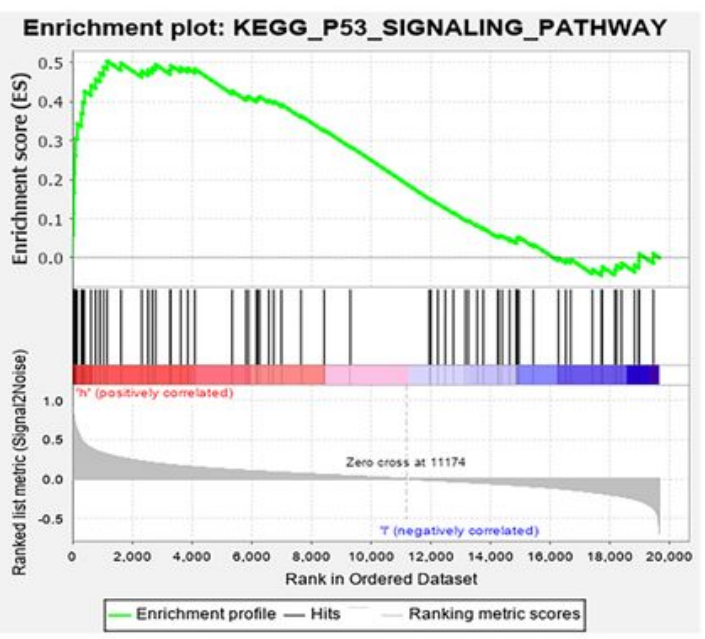

F

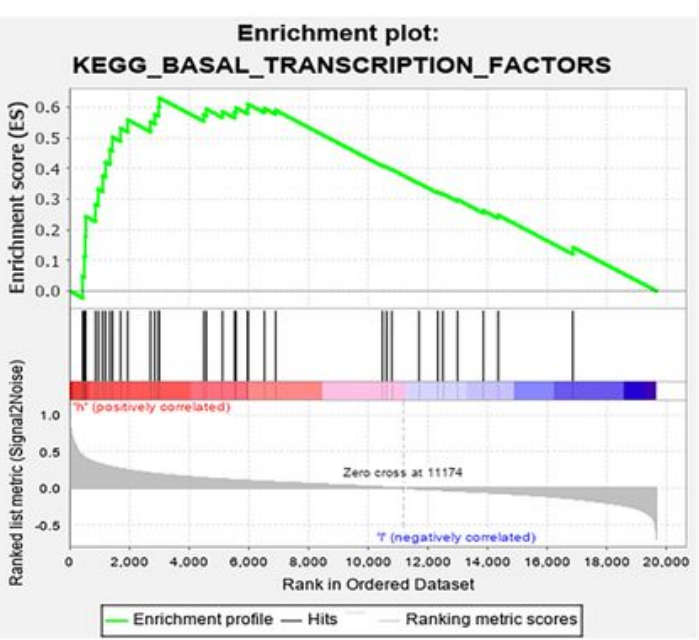

Figure 5

GSEA displayed the main signal pathways derived from high expression of MTFR2 enrichment. Note区 (A) Cell cycle; (B) DNA replication; (C) Homologous recombination; (D) p53 signaling pathway; (E) Oocyte meiosis; (F) Base_excision_repair. 
A

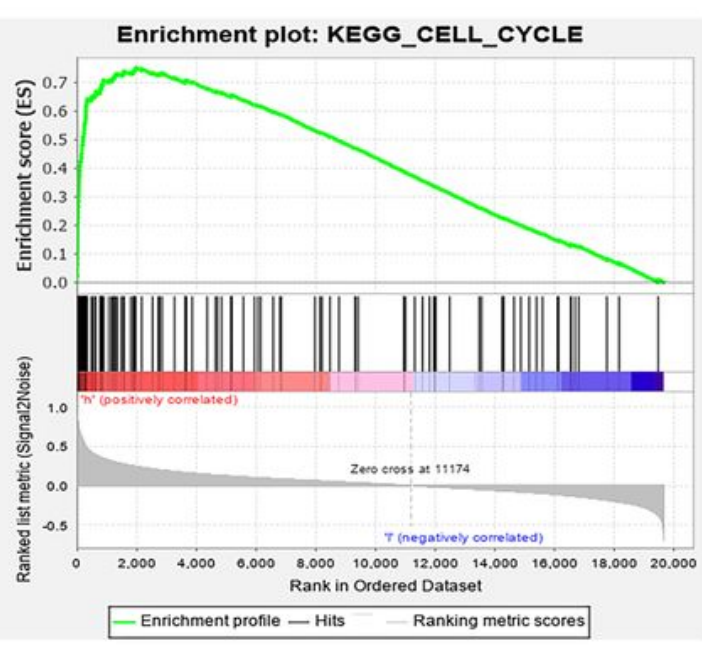

C

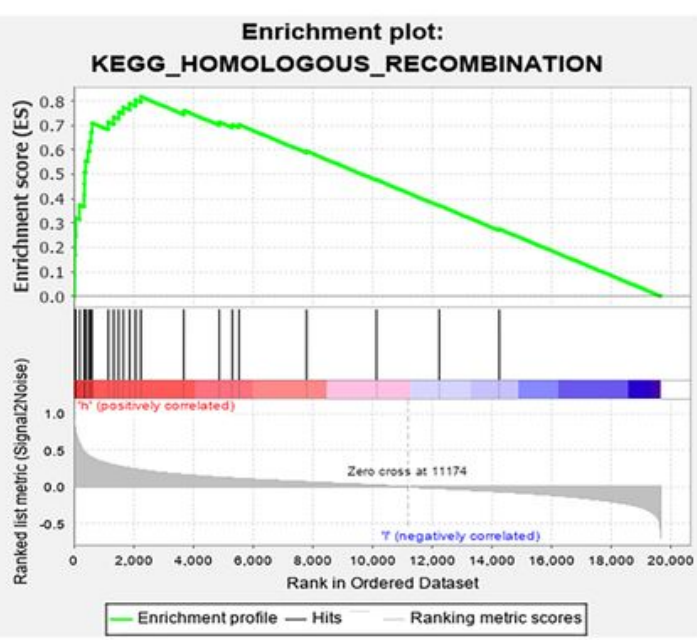

$\mathbf{E}$

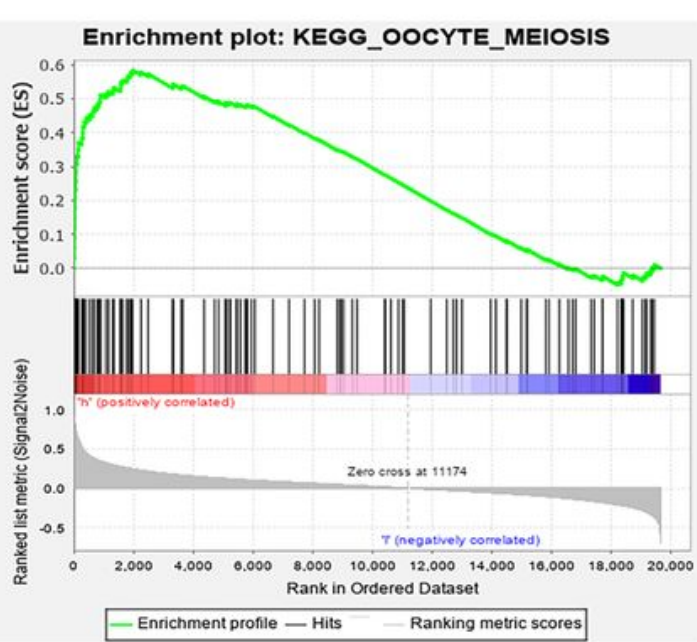

B

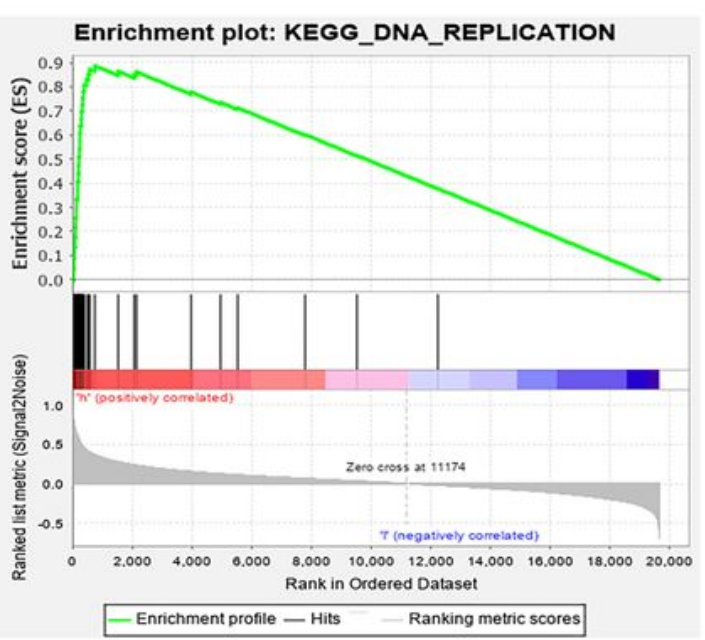

D

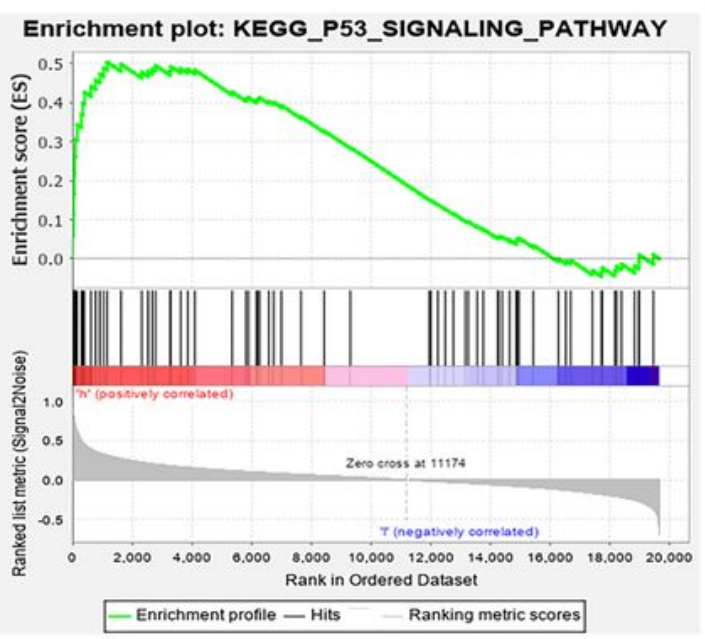

$\mathbf{F}$

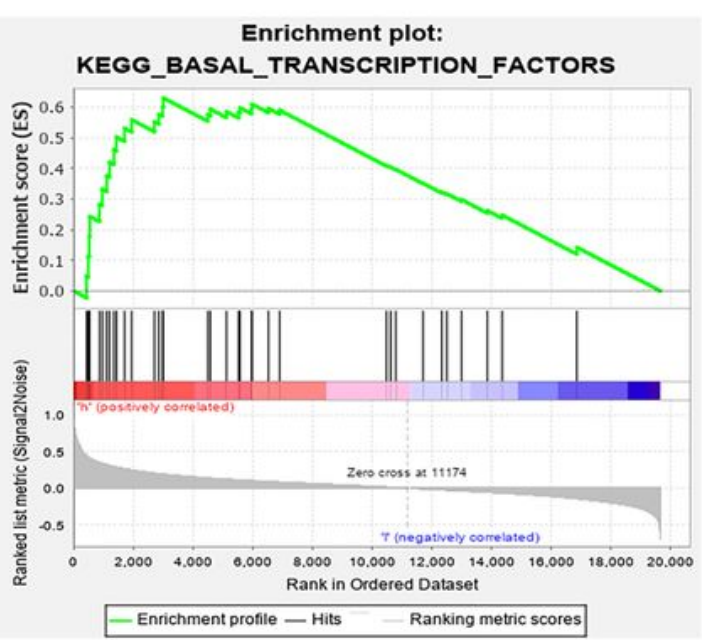

Figure 5

GSEA displayed the main signal pathways derived from high expression of MTFR2 enrichment. Note区 (A) Cell cycle; (B) DNA replication; (C) Homologous recombination; (D) p53 signaling pathway; (E) Oocyte meiosis; (F) Base_excision_repair. 

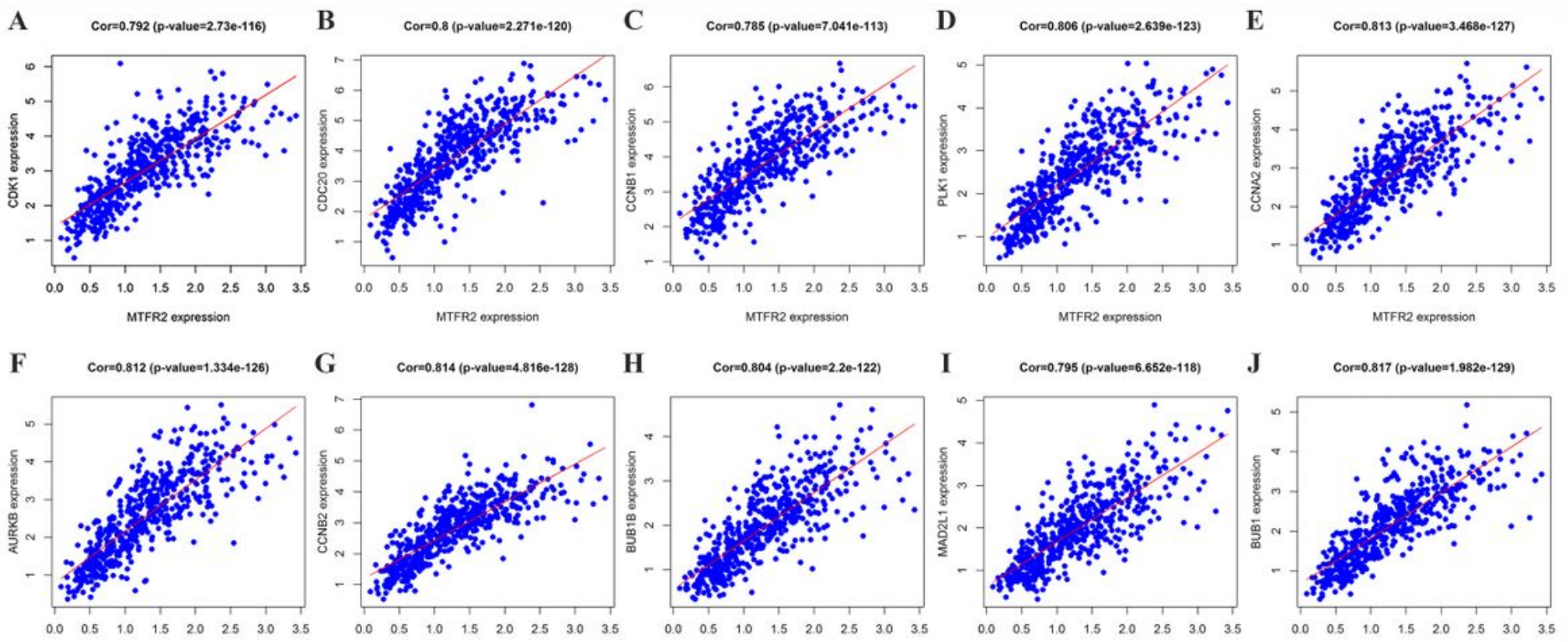

MTFR2 expression
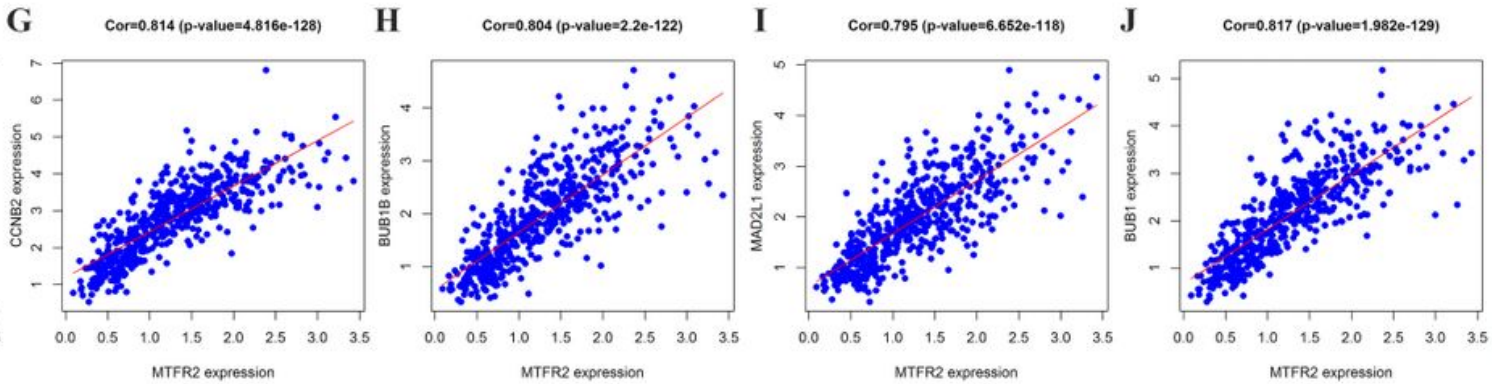

Figure 6

The expression of MTFR2 was correlated to representative top 10 Hub genes. Note $(A) C D K 1 ;(B) C D C 20$; (C) CCNB1; (D) PLK1; (E) CCNA2; (F) AURKB; (G) CCNB2; (H) BUB1B; (I) MAD2L1; (J) BUB1.
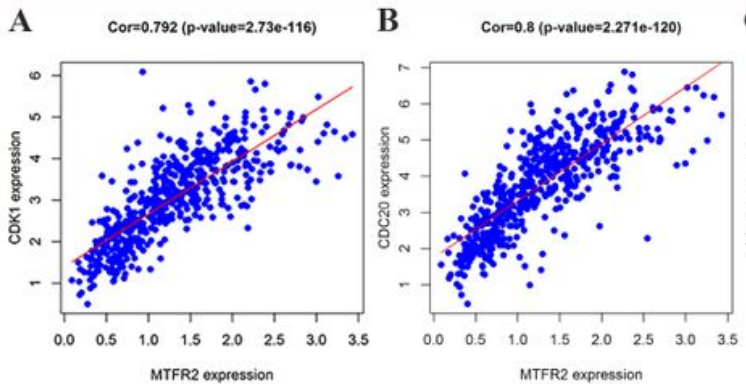

C Cor=0.785 (p-value $=7.041 \mathrm{e}-113)$

D Cor=0.806 (p-value $=2.639 \mathrm{e}-123)$

E Cor=0.813 (p-value=3.468e-127)
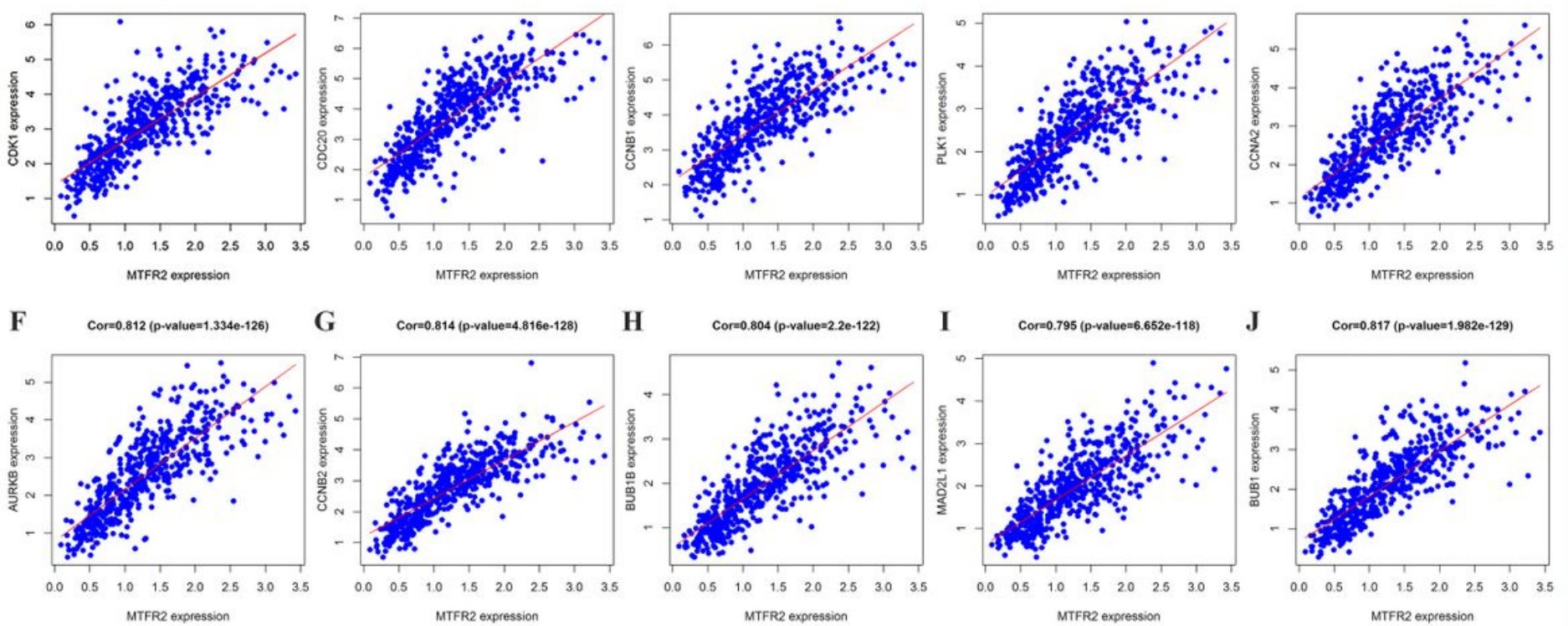

Figure 6

The expression of MTFR2 was correlated to representative top 10 Hub genes. Note $(A) C D K 1$; $(B) C D C 20$; (C) CCNB1; (D) PLK1; (E) CCNA2; (F) AURKB; (G) CCNB2; (H) BUB1B; (I) MAD2L1; (J) BUB1. 


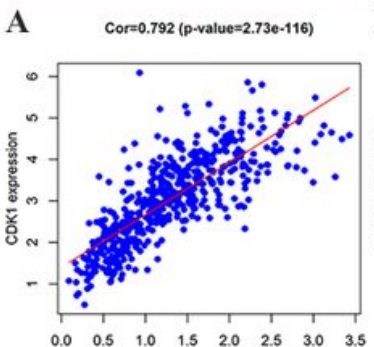

MTFR2 expression

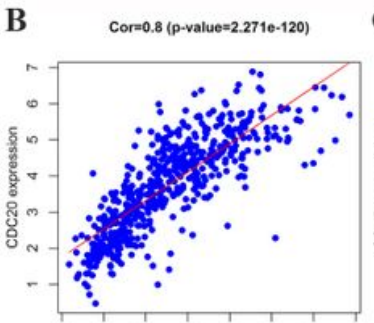

$\begin{array}{llllllll}0.0 & 0.5 & 1.0 & 1.5 & 2.0 & 2.5 & 3.0 & 3.5\end{array}$ MTFR2 expression

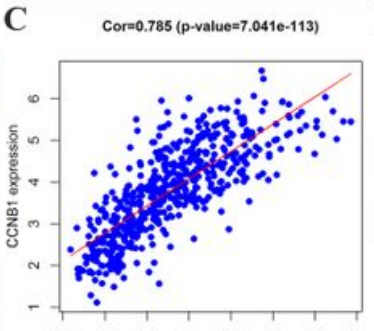

$\begin{array}{llllllll}0.0 & 0.5 & 1.0 & 1.5 & 2.0 & 2.5 & 3.0 & 3.5\end{array}$ MTFR2 expression

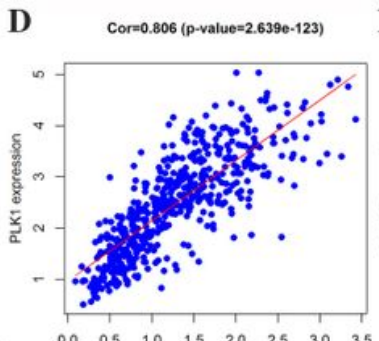

$\begin{array}{llllllll}0.0 & 0.5 & 1.0 & 1.5 & 2.0 & 2.5 & 3.0 & 3.5\end{array}$

MTFR2 expression
E Cor=0.813 (p-value=3.468e-127)

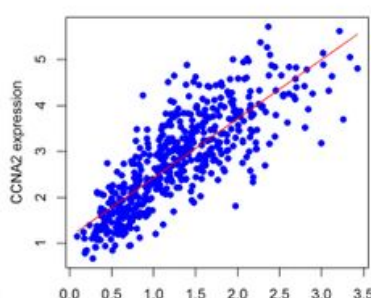

MTFR2 expression

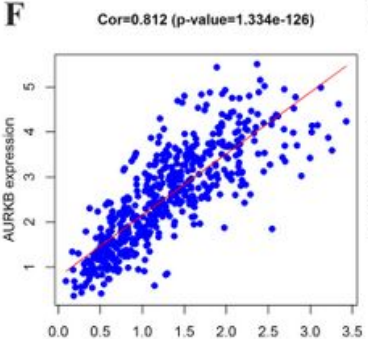

$\begin{array}{llllllll}0.0 & 0.5 & 1.0 & 1.5 & 2.0 & 2.5 & 3.0 & 3.5\end{array}$ MTFR2 expression

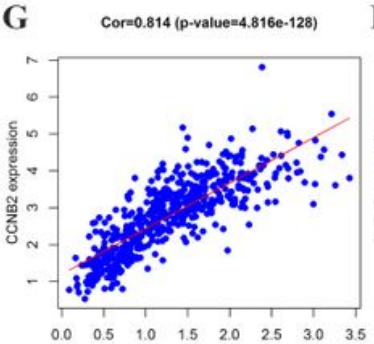

MTFR2 expression
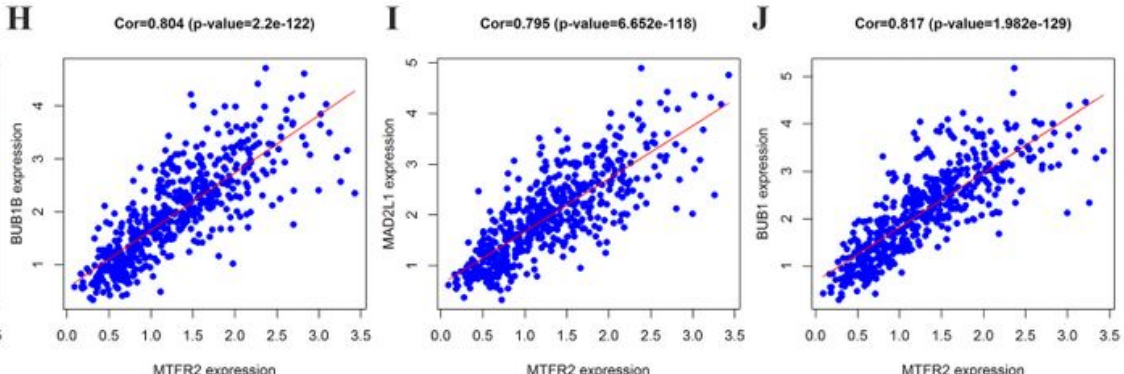

Figure 6

The expression of MTFR2 was correlated to representative top 10 Hub genes. Note $\mathbb{( A )} C D K 1$; (B) CDC20; (C) CCNB1; (D) PLK1;
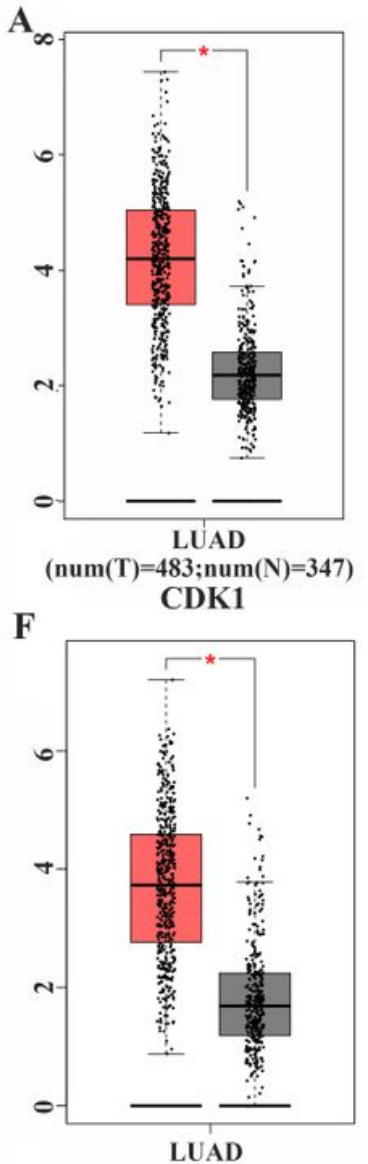

(num $(\mathrm{T})=483 ; \operatorname{num}(\mathrm{N})=347$ ) AURKB
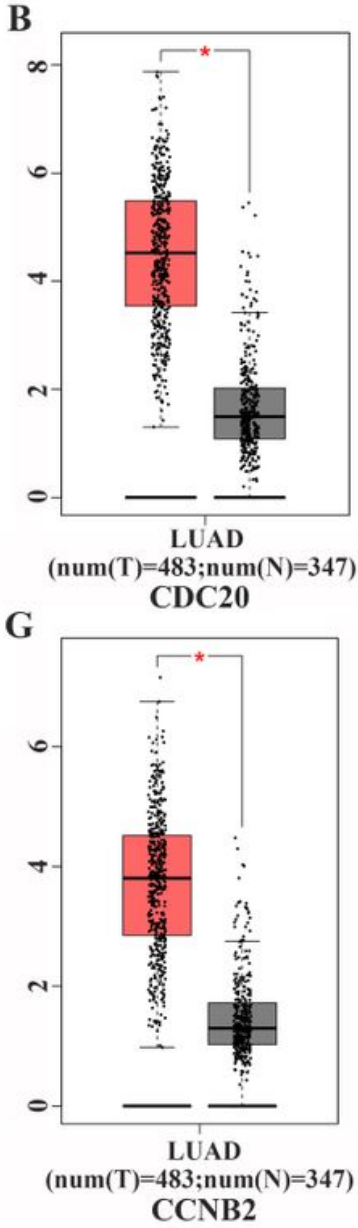

C

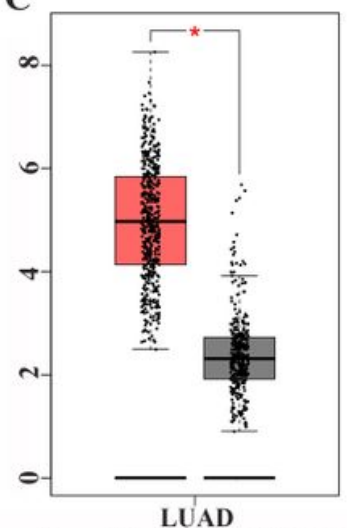

(num $(T)=483 ; \operatorname{num}(N)=347$

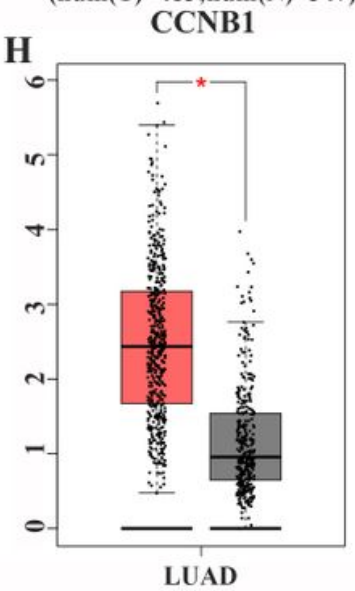

(num $(T)=483$;num $(N)=347$ )

BUB1B
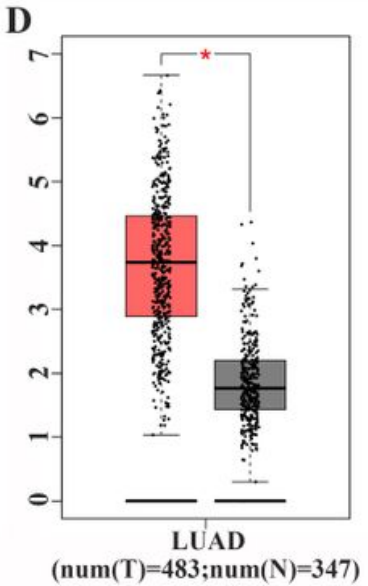

PLK1

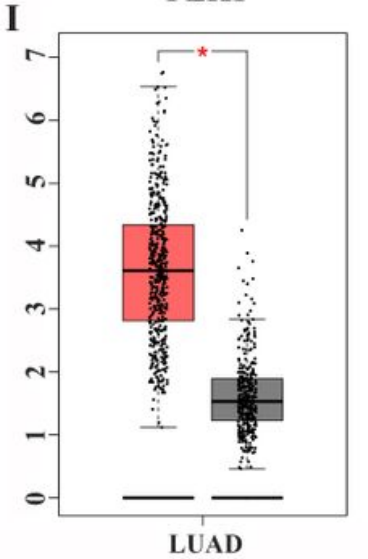

(num(T)=483; num $(\mathrm{N})=347$ ) MAD2L1

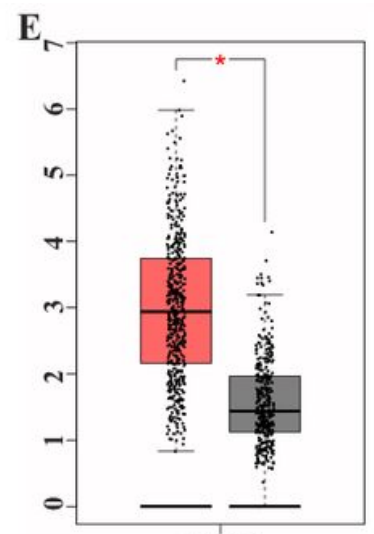

LUAD $(\operatorname{num}(T)=483 ; \operatorname{num}(\mathrm{N})=347)$ CCNA2

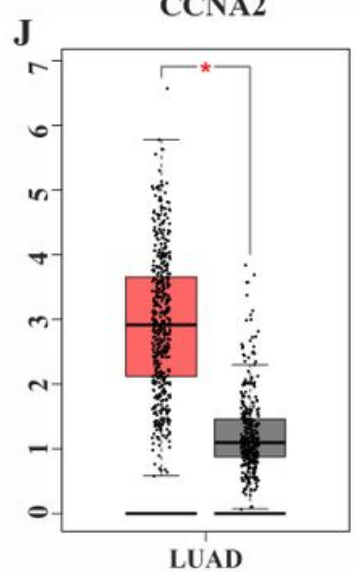

$(\operatorname{num}(\mathrm{T})=483 ; \mathrm{num}(\mathrm{N})=347)$ BUB1

Figure 7 
The expressions of the top10 Hub genes in LUAD tissues from TCGA Database. Note区(A) CDK1; (B) CDC20; (C)CCNB1; (D) PLK1; (E) CCNA2; (F) AURKB; (G) CCNB2; (H) BUB1B; (I) MAD2L1; (J) BUB1. Note: T, LUAD tissue; $N$, normal tissue; red, LUAD tissue; gray, normal lung tissue; ${ }^{*}, p<0.05$.
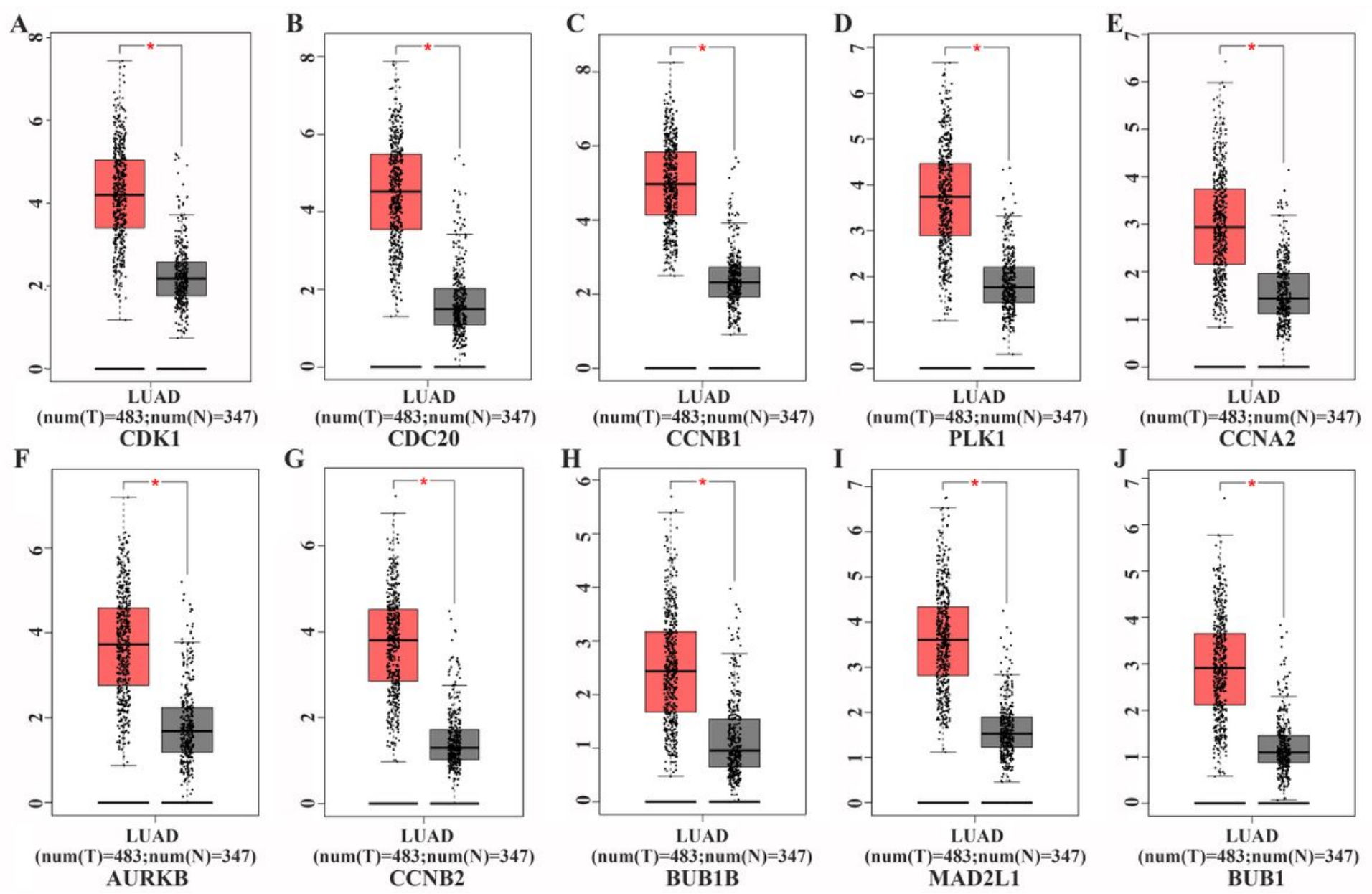

Figure 7

The expressions of the top10 Hub genes in LUAD tissues from TCGA Database. Note $(A) C D K 1 ;(B)$ CDC20; (C)CCNB1; (D) PLK1; (E) CCNA2; (F) AURKB; (G) CCNB2; (H) BUB1B; (I) MAD2L1; (J) BUB1. Note: T, LUAD tissue; $N$, normal tissue; red, LUAD tissue; gray, normal lung tissue; *, $p<0.05$. 

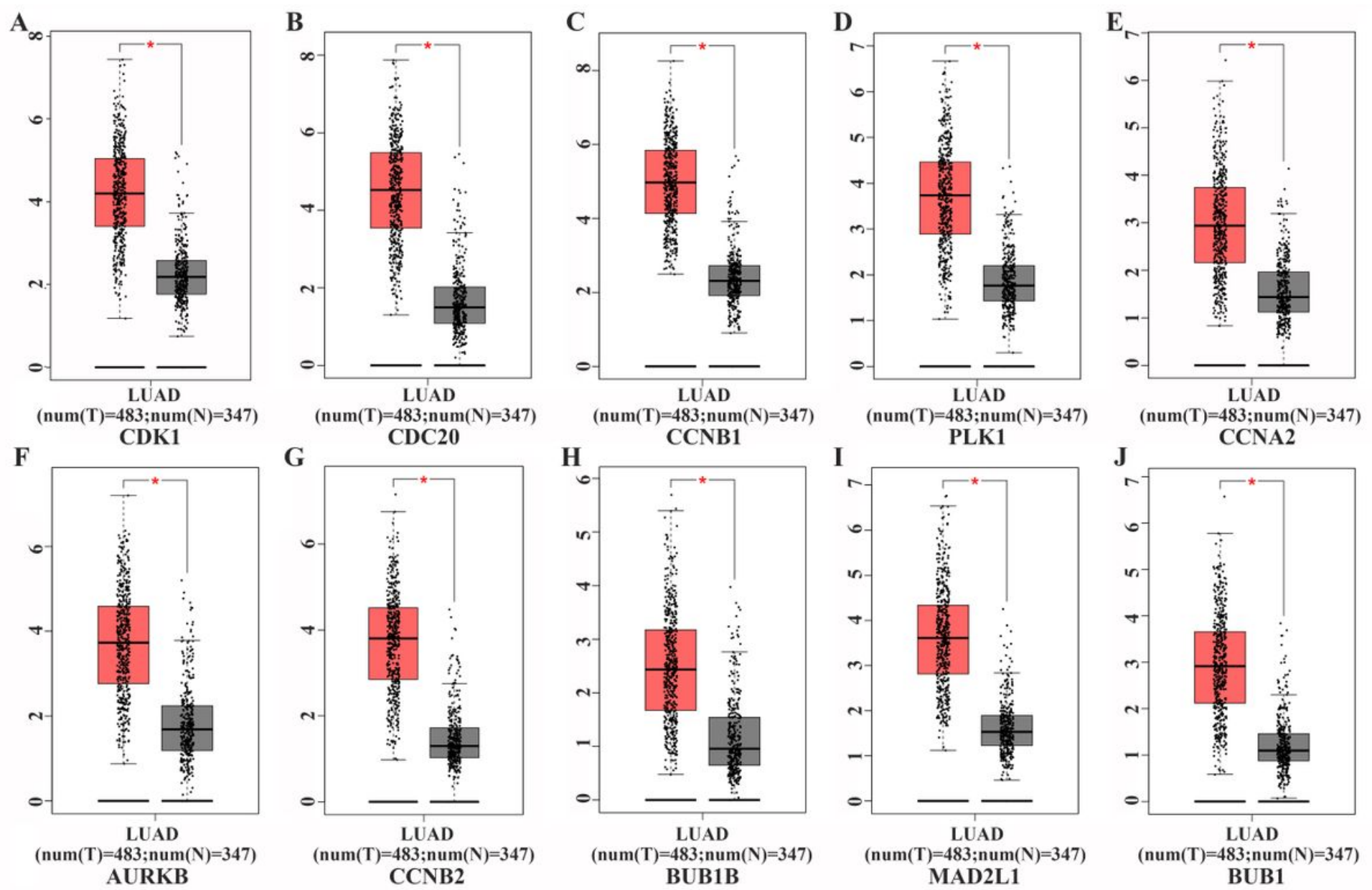

\section{Figure 7}

The expressions of the top10 Hub genes in LUAD tissues from TCGA Database. Note区(A) CDK1; (B) CDC20; (C)CCNB1; (D) PLK1; (E) CCNA2; (F) AURKB; (G) CCNB2; (H) BUB1B; (I) MAD2L1; (J) BUB1. Note: T, LUAD tissue; $N$, normal tissue; red, LUAD tissue; gray, normal lung tissue; ${ }^{*}, p<0.05$. 

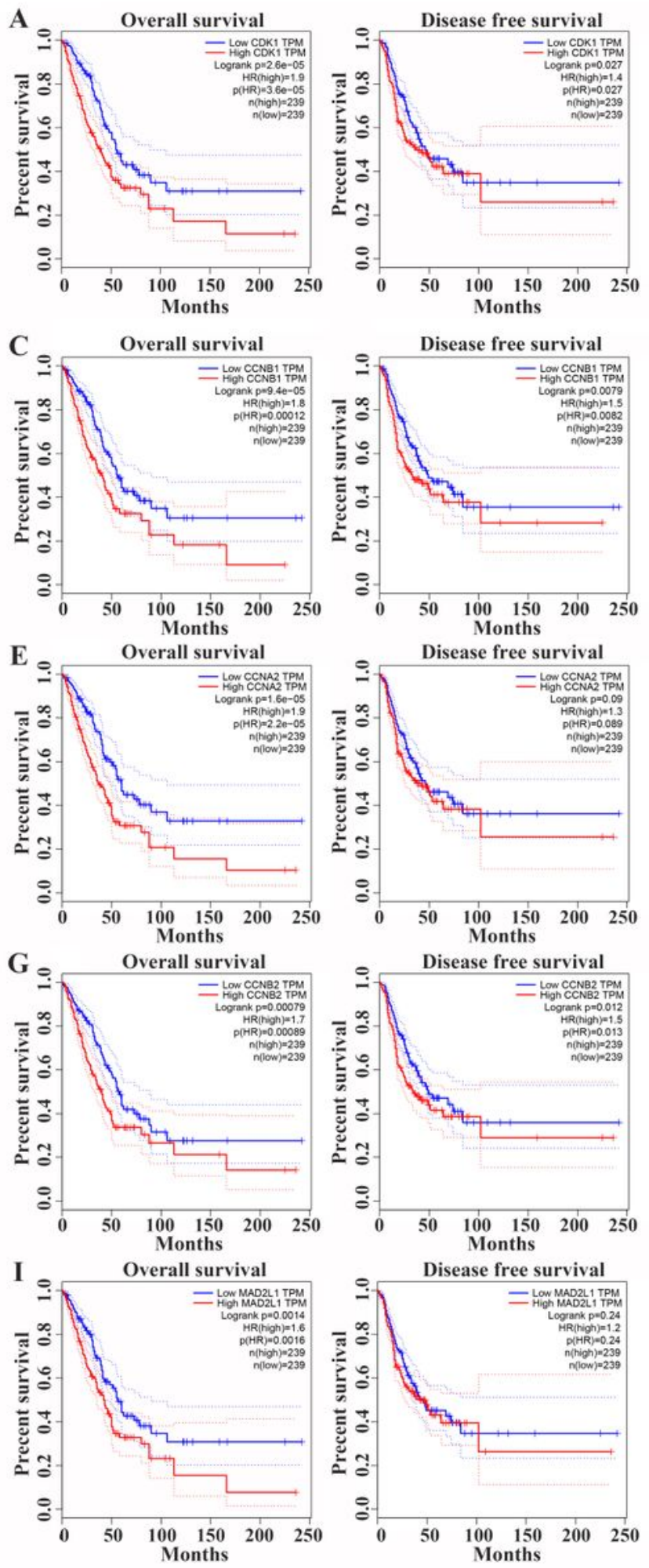
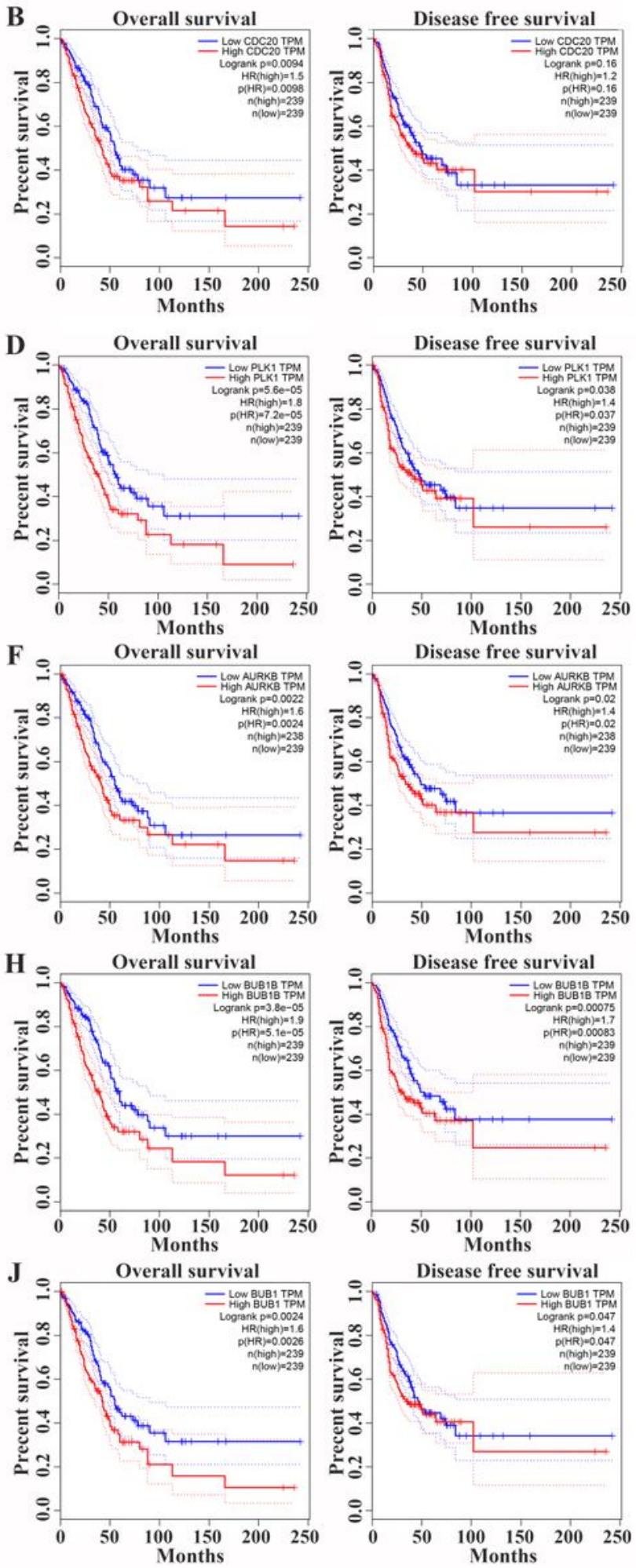

\section{Figure 8}

The value of Hub genes in the prognosis of LUAD from GEPIA. Note $\mathbb{( A )}$ CDK1; (B) CDC20; (C) CCNB1; (D) PLK1; (E)CCNA2; (F) AURKB; (G) CCNB2; (H) BUB1B; (I) MAD2L1; (J) BUB1. 

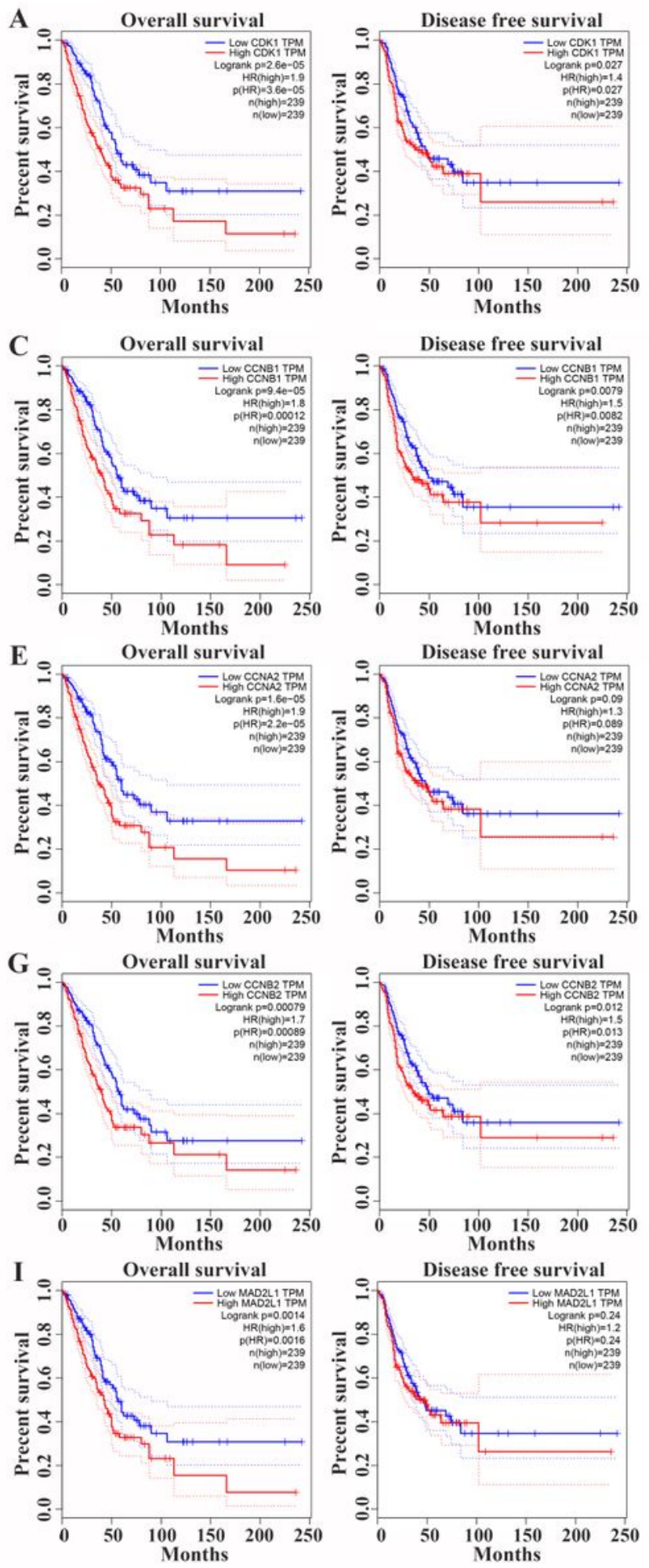
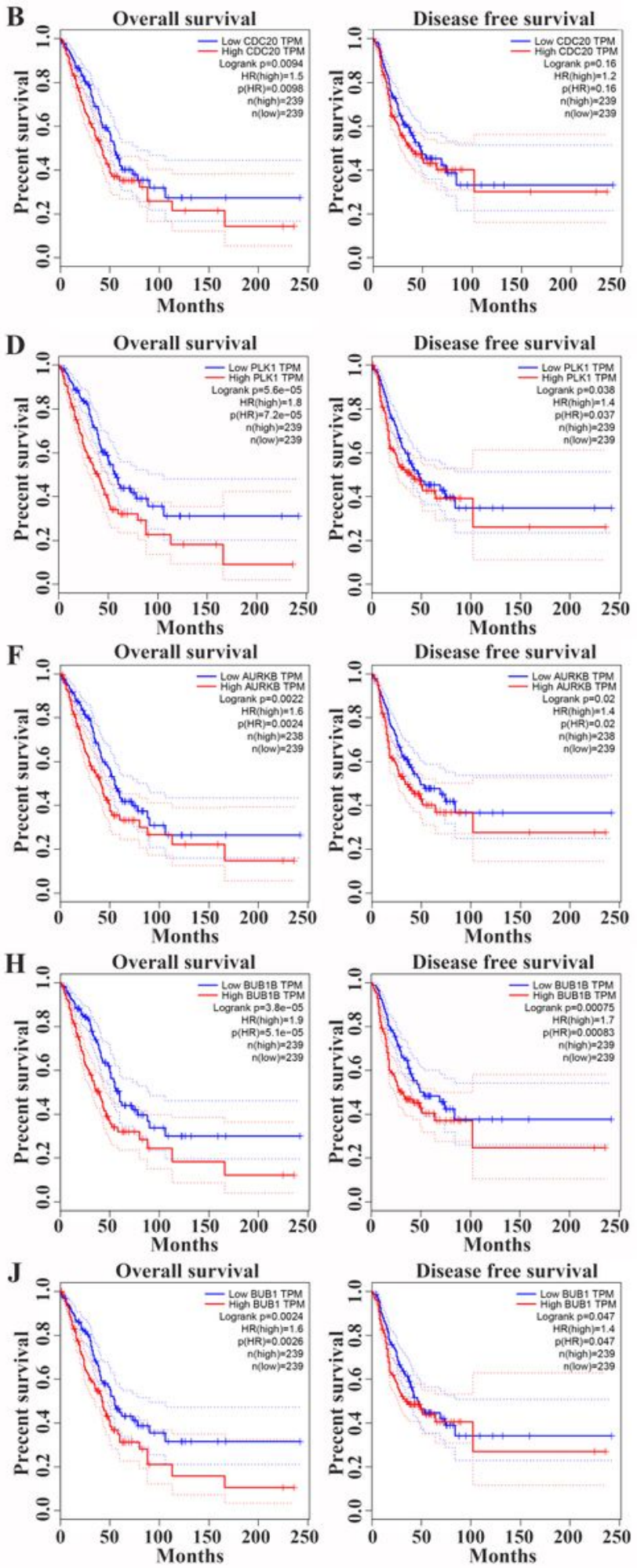

\section{Figure 8}

The value of Hub genes in the prognosis of LUAD from GEPIA. Note $\mathbb{( A )}$ CDK1; (B) CDC20; (C) CCNB1; (D) PLK1; (E)CCNA2; (F) AURKB; (G) CCNB2; (H) BUB1B; (I) MAD2L1; (J) BUB1. 

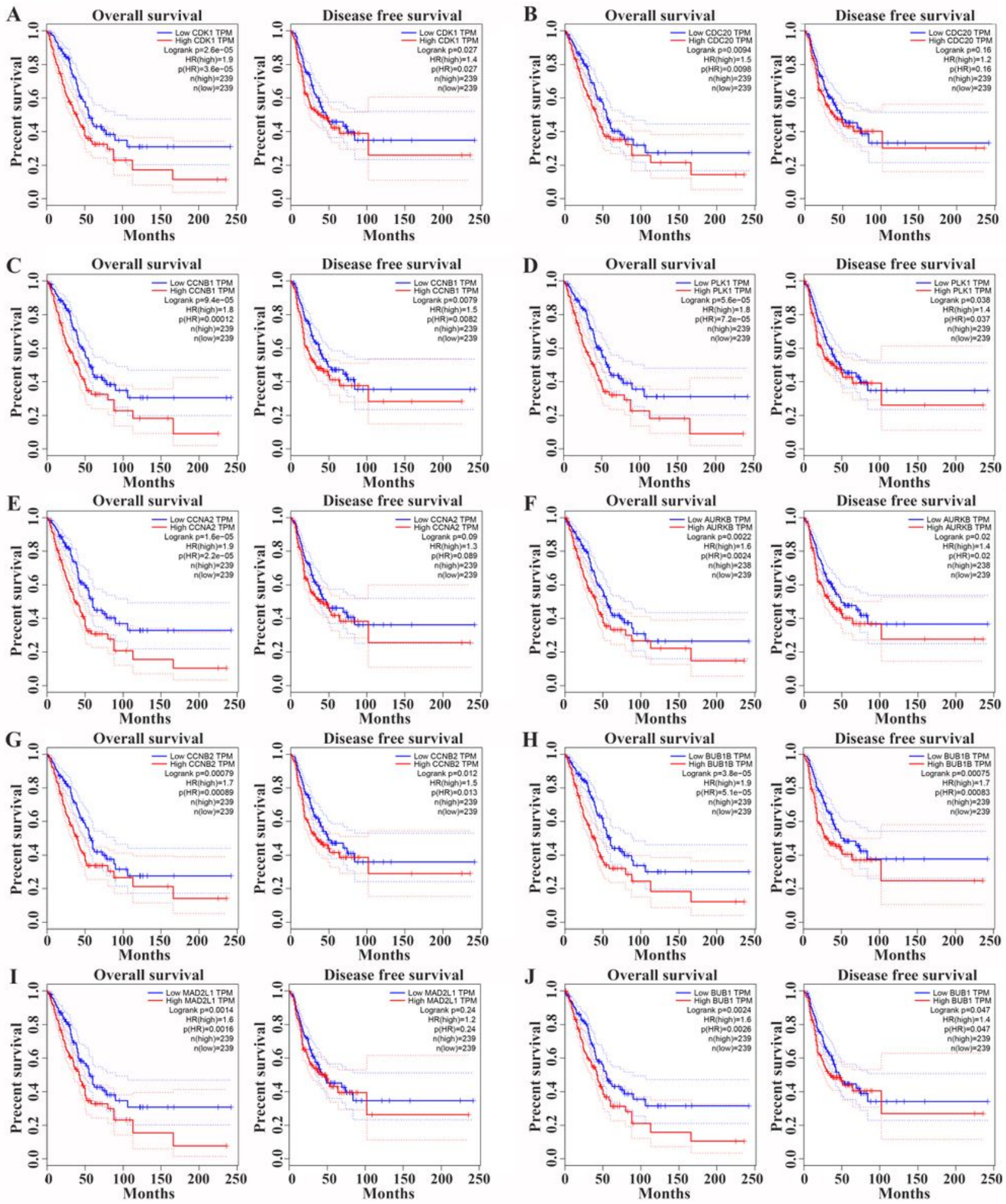

\section{Figure 8}

The value of Hub genes in the prognosis of LUAD from GEPIA. Note区(A) CDK1; (B) CDC20; (C) CCNB1; (D) PLK1; (E)CCNA2; (F) AURKB; (G) CCNB2; (H) BUB1B; (I) MAD2L1; (J) BUB1.

\section{Supplementary Files}


This is a list of supplementary files associated with this preprint. Click to download.

- sfig.docx

- sfig.docx

- sfig.docx

- TableS1.docx

- Tables1.docx

- Tables1.docx

- Tables2.docx

- Tables2.docx

- Tables2.docx 